\title{
Focus plus context visualization based on volume clipping for markerless on-patient medical data visualization
}

\author{
Márcio C. F. Macedo*, Antônio L. Apolinário Jr. \\ Department of Computer Science, Federal University of Bahia (UFBA), Brazil
}

\begin{abstract}
Focus plus context visualization can be used in augmented reality to improve the visual perception of the augmented scene. In the scope of in situ or on-patient medical data visualization, the focus plus context paradigm is used to improve depth perception for physicians showing the patient's anatomy as a focus region in the context of the patient's body. Volume clipping is one technique to realize focus plus context visualization. However, some of the existing methods for focus plus context visualization based on volume clipping do not run in full real time or are prone to artifacts. In this article, we present an extension for two of these techniques to improve performance and image quality of the original approaches. We validate all the techniques in a markerless augmented reality environment. A 3D reference model is tracked by the application, and volumetric medical data are shown to the user at the position of the patient's anatomy. Our technique is able to handle multiple anatomic regions, although the main region of interest used in this article is the face. Moreover, tracking accuracy is improved by the use of a hierarchical approach. From an evaluation of the proposed techniques, the results obtained highlight that all of them are free of artifacts, optimized for real-time performance, and improve the visual quality of the augmented scene.
\end{abstract}

Keywords:

Volume clipping, Focus plus context visualization, Augmented reality, Volume rendering

\section{Introduction}

2 Physicians see medical data, typically images of a patient's 3 anatomic structures, on a monitor and they must analyze and ${ }_{4}$ mentally compose what is shown on the screen. This mental ${ }_{5}$ model of the patient's anatomy will help the physician provide ${ }_{6}$ health care in time-critical situations. Therefore, the physician 7 must have sufficient knowledge of the patient's and general hu8 man anatomy to proceed appropriately during any medical pro9 cedure (e.g., diagnosis, surgery). With the availability of aug10 mented reality (AR) technology, one can take over this task of 11 mental mapping by transferring it to a computer. Therefore, 12 the physician will be able to visualize, at the same time, the ${ }_{13}$ patient and a part of the patient's anatomy. On-patient or in ${ }_{14}$ situ medical data visualization can be used to improve surgical 15 planning, training, medical diagnosis, and post-operative exam${ }_{16}$ ination. This kind of application is desirable in fields such as 17 those involving craniofacial data, in which the visualization of ${ }_{18} 3 \mathrm{D}$ examinations on the patient may help the physician under19 stand the trauma.

20 AR is a technology which augments the view of a real scene ${ }_{21}$ with additional virtual information. Accurate tracking of the 22 real scene, realistic rendering of the virtual data, and real-time ${ }_{23}$ user interactivity are the most important technical challenges of

\footnotetext{
${ }^{*}$ Corresponding author

Email addresses: marciocfmacedo@gmail . com (Márcio C. F. Macedo), apolinario@dcc.ufba.br (Antônio L. Apolinário Jr.)
}

${ }_{24} \mathrm{AR}$ applications [1]. The face is a part of the body in which ${ }_{25}$ depth- or texture-based tracking is easier because of the avail${ }_{26}$ ability of face detection algorithms and the presence of distin${ }_{27}$ guishable geometric structures. We take advantage of this to fo28 cus on the problem of on-patient medical data visualization for ${ }_{29}$ patients with craniofacial traumas. The decision to use a mark${ }_{30}$ erless AR (MAR) environment for tracking resulted from ob${ }_{31}$ servations of the current limitations of the techniques proposed ${ }_{32}$ in the field of on-patient medical data visualization. Here, we ${ }_{33}$ are mainly interested in investigating the possibility of devel${ }_{34}$ oping an MAR environment for on-patient medical data visu${ }_{35}$ alization which supports high-quality on-patient visualization ${ }_{36}$ and depth-based tracking (invariant to illumination conditions). ${ }_{37}$ Taking advantage of our main motivation to improve the physi38 cian's knowledge of the patient with craniofacial trauma, in this 39 work we focused our tests on the patient's head as the region 40 of interest (ROI). Although we have developed a solution for ${ }_{41}$ the scenario of craniofacial data visualization, in this article we 42 show how the MAR environment can be adapted for other pa43 tient ROI (i.e., torso and pelvis; Section 6). The generality of ${ }_{44}$ the proposed work is discussed in this article.

45 Traditionally, on-patient medical data visualization applica${ }_{46}$ tions superimpose virtual medical data on the patient. However, ${ }_{47}$ in such applications, the virtual content seems to be floating in 48 front of the patient. As stated in previous work [2, 3, 4, 5], a ${ }_{49}$ better solution is to show the patient's anatomy as a focus re${ }_{50}$ gion in the context of the patient's own body. This process is ${ }_{51}$ known as focus plus context $(\mathrm{F}+\mathrm{C})$ visualization paradigm [6], 
52 and it is known to improve the visual perception of the con- 104 53 tent being visualized. In the field of volume rendering, one way 105 54 to improve the understanding and extend the exploration of the 106 55 medical volume is by use of volume clipping. Therefore, the ${ }_{56}$ effect of volume clipping added in an $\mathrm{F}+\mathrm{C}$ visualization tech${ }_{57}$ nique is a new tool for the user to explore and understand the 58 augmented scene.

59 The existing techniques for $\mathrm{F}+\mathrm{C}$ visualization based on vol60 ume clipping are prone to artifacts or do not run in full real ${ }_{61}$ time [5]. Such issues decrease the application's visual quality 62 and performance, respectively. One way to solve both problems ${ }_{63}$ is by use of an adaptive strategy to mitigate artifacts and shaders 64 to execute the technique in parallel.

65 In this article, we present improvements in terms of perfor${ }_{66}$ mance and visual quality over the $\mathrm{F}+\mathrm{C}$ visualization techniques ${ }_{67}$ based on volume clipping proposed in [5]. We expand the eval68 uation of the MAR environment for different ROI in the patient 69 and improve tracking accuracy through the use of a hierarchical 70 algorithm. A more detailed description of the algorithms used 71 in the entire solution (i.e., MAR environment and $\mathrm{F}+\mathrm{C}$ visual72 ization) and an in-depth analysis of the results obtained and the ${ }_{73}$ limitations of the proposed approach are presented as well.

The remainder of this article is organized as follows. Sec75 tion 2 reviews recent related work on medical AR and $\mathrm{F}+\mathrm{C}$ 76 visualization applied in AR. Section 3 introduces the MAR en77 vironment used in this article for validation of the $\mathrm{F}+\mathrm{C}$ tech78 niques. Section 4 presents the $\mathrm{F}+\mathrm{C}$ techniques based on vol79 ume clipping for on-patient medical data visualization. Section ${ }_{80} 5$ presents the tests conducted and the experimental results ob${ }_{81}$ tained. Section 6 discusses the results obtained and the limita82 tions of this work. In Section 7, a summary of the article and 83 recommendations for future work are presented.

\section{${ }_{84}$ 2. Related work}

85 Medical AR systems for on-patient medical data visualiza${ }_{86}$ tion have been driven by different approaches in recent years. ${ }_{87}$ In this section, we classify the approaches on the basis of their ${ }_{88}$ tracking technology: marker based or markerless.

${ }_{89}$ Over the past decades, many relevant approaches have been ${ }_{90}$ proposed for marker-based medical AR, such as those in [3, 4, ${ }_{91}$ 7]. Artificial fiducial markers provide fast and accurate tracking 92 because of their shape; however, they are commonly associated ${ }_{93}$ with some issues which make this technology unsuitable for ${ }_{94}$ on-patient medical data visualization applications:

- They are intrusive, because they are not part of the original scene.

- When the traditional fiducial marker, such as the one used in popular applications such as ARToolKit [8], is not used, the optical tracking system hardware may be too expensive.

- In general, this kind of tracking must operate only on the image space, according to features computed from the pixels. The main drawbacks for this color- or texture-based tracking are the susceptibility to illumination conditions and marker occlusion, which may affect the accuracy of the tracking algorithm.

Recently, systems have been proposed in the field of marker108 less medical AR. Some of them do not run in real-time (more 109 than 15 frames per second, FPS) [9, 10] and others rely on 10 specific prior knowledge about the ROI to be tracked (see $111[11,12,13]$ for the body and [14] for the face). To the best of 112 our knowledge, there is only one exception which can be used ${ }_{113}$ for general-purpose markerless on-patient medical data visual114 ization: the semiautomatic approach proposed in [15, 16, 17].

115 The semiautomatic MAR environment uses an RGB-D sen116 sor to reconstruct and track a 3D reference model of the pa117 tient's ROI through the AR live stream. Then, after the virtual 118 medical data positioning, it can be displayed for a physician 119 at the location of the patient's real anatomy. Real-time perfor120 mance is achieved by exploitation of the parallelism provided 121 by the graphics processing unit (GPU).

122 To validate the $\mathrm{F}+\mathrm{C}$ visualization techniques, we use a ${ }_{123}$ marker-free tracking algorithm because it requires a low pro124 cessing time and can operate on customer hardware with good 125 accuracy. A first necessary step is to evaluate the performance 126 and visual quality of the proposed approach. In this sense, the 127 semiautomatic MAR environment proposed in $[15,16,17]$ is 128 used because it runs in real time and, with some adaptations, its 129 tracking solution can be applied for several ROI in the patient, 130 in contrast to other state-of-the-art solutions. Such adaptations 131 are discussed in Section 6.

132 An application for on-patient medical data visualization re${ }_{133}$ quires special attention to be paid to the composition of the vir${ }_{134}$ tual and real entities of the AR environment. Recently, many 135 approaches have been proposed in the field of $\mathrm{F}+\mathrm{C}$ visual${ }_{136}$ ization to dynamically define how this composition should be ${ }_{137}$ done. These, also known as ghosting or X-ray vision tech138 niques, share the concept of an importance map, a mask (similar 139 to an alpha mask) which controls how real and virtual entities 140 should be blended.

Sandor et al. [18] designed a method for importance map 142 computation based on the feature regions of both real and vir${ }_{143}$ tual objects inspired by three features: luminosity-to preserve ${ }_{144}$ regions with high illumination; hue-to preserve strong colors; 145 motion-to preserve moving structures in the final rendering. ${ }_{146}$ As stated by Sandor et al. [18], this work was an extension of 147 the work of [19], which is based on edge overlay to improve 148 spatial perception.

149 Mendez et al. [20] proposed an $\mathrm{F}+\mathrm{C}$ technique in which 150 the lightness and color contrast of a given image are modified 151 according to the importance map computed from a live color 152 video. By adding subtle changes in the image, they guarantee 153 temporal and spatial coherence between frames. The problem 154 with this approach is its performance, which does not achieve 155 the full 30 FPS even when it is implemented on the GPU.

156 An adaptive $\mathrm{F}+\mathrm{C}$ visualization technique was recently intro${ }_{157}$ duced by Kalkofen et al. [21]. In their approach, an importance ${ }_{158}$ map is computed for the occluder [20] and the occludee is in159 serted into the scene. Then, another importance map is com- 
160 puted from this resulting image and is then compared against ${ }_{161}$ the first map computed. Regions on the first importance map 162 that are not present in the final rendering are then emphasized ${ }_{163}$ to be visible. This approach improves the visual quality of the 164 augmented scene and it runs in real time. However, it is not suit165 able for MAR environments, as it alone requires a processing ${ }_{166}$ time of $33 \mathrm{~ms}$. Therefore, this additional time would decrease ${ }_{167}$ severely the performance of an MAR application.

${ }_{168} \mathrm{~F}+\mathrm{C}$ rendering was also proposed for visualization of under169 ground structures in street scenes [22, 23, 24]. In these ap170 proaches, a method is used to dynamically compute when the 171 underground structures must be rendered in relation to moving 172 objects present in the scene. Although the final visual quality is 173 good, the performance of the existing techniques is not full real 174 time.

175 Traditional methods which compute the importance maps 176 from live color video of the real scene are prone to errors be177 cause they are dependent on the illumination and material prop178 erties of the real environment. To overcome these problems, 179 Mendez and Schmalstieg [25] proposed a method to compute 180 an importance mask based on the 3D model of the scene. This ${ }_{181}$ task is accomplished by use of techniques such as mesh saliency ${ }_{182}$ [26] or through user interaction in a preprocessing step. The ${ }_{183}$ problem with this approach is that the importance mask cre184 ation requires some processing time. Therefore, the user can185 not change interactively the importance mask during an AR live 186 stream.

${ }_{187}$ The methods proposed in the literature for $\mathrm{F}+\mathrm{C}$ visualization ${ }_{188}$ in general AR applications capture the features of the image; 189 however, their importance maps are not accurate enough to be 190 used for medical applications.

$191 \mathrm{~F}+\mathrm{C}$ visualization has been proposed not only for AR, but 192 also for volume rendering. In this case, it is used to define how 193 the internal structures of the volume (e.g., bone, organ) should 194 be visualized in the context of the soft tissue.

195 Bruckner et al. [27, 28] proposed a method for context196 preserving volume rendering. From factors such as shading in197 tensity, gradient magnitude, distance to the eye point, and pre198 viously accumulated opacity, the method allows the user def199 inition of the $\mathrm{F}+\mathrm{C}$ rendering according to only two parame200 ters which controls these four factors to interactively change 201 the transparency level between internal and external structures 202 of the volume. The technique is easy to implement and runs 203 directly on the shader. An extension of this algorithm was pro204 posed by [29]. It incorporates rotation, scale, position, and 205 mouse click to dynamically select focus and context regions.

206 Kruger et al. [30] proposed ClearView. Four layers (i.e., fo207 cus and context structures, isosurface's normal and curvature) 208 are generated and composed for each frame in order to define 209 the final visualization. The main disadvantages of this method ${ }_{210}$ is that it is naturally multi-pass (i.e., one shading pass is re${ }_{211}$ quired to compute every layer) and the layers must be recom${ }_{212}$ puted for every change of viewpoint. Therefore, the approach ${ }_{213}$ has a considerable cost in terms of performance.

${ }_{214}$ Kirmizibayrak et al. [31] proposed a volumetric brush 215 method for interactive definition of focus and context regions ${ }_{216}$ for volumetric models. Their approach runs in real time and
217 provides a good alternative for physicians to visualize medical ${ }_{218}$ data, especially for applications such as radiation therapy.

${ }_{219} \mathrm{~F}+\mathrm{C}$ solutions have also been proposed in the literature to 220 help in the visualization of complex fiber distributions [32], 221 blood flow [33], structured biomedical data [34] and ultrasound 222 [35].

${ }_{223}$ The main goal of the $\mathrm{F}+\mathrm{C}$ visualization techniques applied 224 in AR environments is to improve the depth perception of the 225 augmented scene. This is specially important for medical AR 226 applications, in which physicians must have good understand227 ing of the augmented scene to proceed with their tasks appropri228 ately. Despite the number of techniques and applications which 229 have been proposed for medical AR, only a few of them con230 sider the visualization a relevant aspect for the application [36]. ${ }_{231}$ We show here some of the visualization techniques proposed ${ }_{232}$ specifically for the field of medical AR to achieve the goal of 233 improved depth perception.

${ }_{234}$ Lerotic et al. [37] suggested the use of a $p q$ space-based 235 nonphotorealistic rendering method for augmented visualiza236 tion in minimally invasive surgery. In their approach, the ${ }_{237}$ anatomic surface is expressed in terms of a $p q$-space represen238 tation, where $p$ and $q$ are the slope of the surface along the $x$ 239 and $y$ axes. These values are used to determine which regions 240 of the suface are more salient and must be emphasized in the ${ }_{241}$ final rendering. Pratt et al. [38] extended this technique to run 242 in real time on the GPU. For it to do so, the original algorithm 243 was simplified by use of an intensity gradient filter to highlight 244 anatomic surface details.

Bichlmeier et al. [39] proposed the virtual mirror, a tech246 nique which improves not only the depth perception, but also 247 the navigation, visualization, and understanding of the virtual 248 structures positioned into the augmented scene. This can be 249 achieved through the use of a specialized hardware setup and 250 standard techniques in computer graphics for mirror reflection 251 computation.

252 Kersten-Oertel et al. [40] provided an evaluation of several ${ }_{253}$ strategies for improving depth perception (namely, fog, pseu254 dochromadepth, kinetic depth, edge depiction, and stereo) in 255 the medical data visualization. The evaluation was conducted 256 with novice and expert users, and the conclusion was that the 257 fog and pseudochromadepth [41] techniques improve under258 standing of the medical structures.

259 One of the first techniques proposed for $\mathrm{F}+\mathrm{C}$ visualization in 260 the field of on-patient medical data visualization was the con261 textual anatomic mimesis (CAM) proposed by Bichlmeier et 262 al. [2]. Its importance map is defined by three parameters: the ${ }_{263}$ curvature of the patient's skin surface, the angle of incidence 264 (i.e., angle between the normal on the skin surface and a vector 265 pointing from the position of the surface and the eye), and the 266 distance falloff (i.e., the distance between each point on the sur267 face and the intersection point of the line of sight and the skin 268 surface). Differently from the color-based methods mentioned 269 for the $\mathrm{F}+\mathrm{C}$ techniques applied in the common AR scenario, 270 this one operates directly on the shader and is not dependent 271 on illumination or texture for the importance map definition. 272 Although it provides improved visualization of the 3D medical ${ }_{273}$ data in the scene, it does not give special attention to the effect 
274 of volume clipping.

275 Aiming to provide the physician with more tools to improve 276 the visual perception of the augmented scene, previous work 277 has proposed three $\mathrm{F}+\mathrm{C}$ techniques based on volume clipping: 278 the smooth contours technique, and the visible background on ${ }_{279} \mathrm{CT}$ and MRI data techniques [5]. Each of them defines a spe280 cific region of the volume to be used as a focus or context re${ }_{281}$ gion. However, the smooth contours technique is not optimized 282 for real-time performance, and the visible background on MRI ${ }_{283}$ data technique generates images with visible artifacts and, in 284 fact, it was not evaluated with respect to visual quality, although 285 it showed promising results. In this article, we present an ex286 tension of the work of [5] to solve such problems.

\section{3. Markerless augmented reality environment}

${ }_{288}$ In this section, we describe the MAR environment used in 289 this work, which is mostly based on the one proposed in [15, $29016,17]$. However, we present modifications to improve tracking 291 accuracy while enabling real-time performance for the MAR 292 environment.

293 An overview of the proposed solution is given in Figure 294 1. First, we reconstruct a 3D reference model of the pa295 tient's ROI to track it without markers in the AR live stream. 296 Three-dimensional (3D) reference model reconstruction re297 quires markerless tracking to align the different viewpoints ac298 quired from the patient's ROI. In contrast, markerless track299 ing requires $3 \mathrm{D}$ reference model reconstruction to perform live 300 tracking during the on-patient medical data visualization. Be301 cause of the recent availability of MAR environments for on302 patient medical data visualization, which are based on off-the303 shelf hardware and provide good composition of the real and 304 virtual entities in the $\mathrm{AR}$ environment $[17,14,13]$, they can 305 be used to validate the $\mathrm{F}+\mathrm{C}$ techniques. From the estimated 306 camera pose (i.e., position and orientation), the medical vol307 ume can be rendered and displayed for a physician inside the 308 patient's body at the location of the real anatomy. Volume 309 data are rendered according to standard volume rendering tech310 niques. After volume rendering, $\mathrm{F}+\mathrm{C}$ visualization techniques 311 (i.e., smooth contours technique, and visible background on ${ }_{312} \mathrm{CT}$ and MRI data techniques) are used to define which parts ${ }_{313}$ of the volume will be visualized in the final augmented scene. ${ }_{314}$ Real-time performance is achieved by implementation of the 315 MAR environment (i.e., markerless tracking and 3D reference 316 model reconstruction) on the general-purpose GPU and vol317 ume rendering together with the $\mathrm{F}+\mathrm{C}$ visualization using GLSL 318 shaders.

${ }_{319}$ To track the medical volume in the AR environment without 320 markers, a 3D reference model of the patient's ROI is generated. ${ }_{321}$ To reconstruct a single 3D reference model of the patient's ROI, ${ }_{322}$ it is necessary to detect it and segment it from the real scene 323 captured by the RGB-D sensor. In this work, F+C visualization 324 techniques were validated in a scenario where the ROI consists 325 mainly of the patient's face. For face detection and segmenta326 tion, the Viola-Jones face detector [42] is applied in the color 327 image provided by the RGB-D sensor. Once the ROI has been ${ }_{328}$ segmented in the color image, this segmented region is fixed.

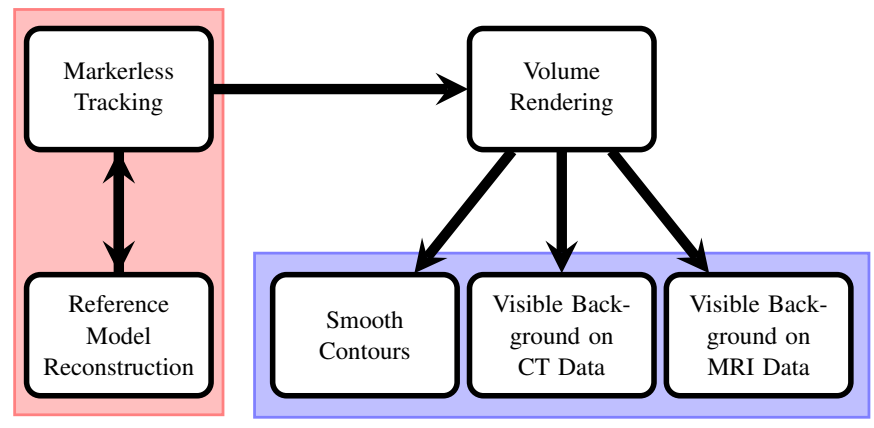

Markerless Augmented Reality Environment

Focus + Context Visualization

Figure 1: Integrated solution for markerless on-patient medical data visualization based on focus plus context rendering viewed as components and their relationships.

${ }_{329}$ Then, the user is constrained to move the ROI in this fixed ззо region so that the system can capture the different viewpoints ${ }_{331}$ from the same ROI. Through calibration of the color and depth зз2 sensors, it is possible to transfer this segmented region of the ззз color image to the depth image. The depth map is denoised with 334 a bilateral filter [43], and then the pyramid algorithm is applied 335 to build low-resolution approximations of the original denoised ${ }_{336}$ depth map [44, 45]. To do so, a mean filter is implemented on 337 the GPU to compute only two coarse levels from the denoised ${ }_{338}$ depth map. Filtered depth maps are converted into vertex and ззя normal maps. Maps computed from the original depth map are 340 used through all the steps of the algorithm. Coarse vertex and 341 normal maps are used only for tracking. Then, the KinectFu342 sion algorithm [46] is used to reconstruct the reference model 343 of the patient's ROI in real time.

KinectFusion is an algorithm that reconstructs high-quality ${ }_{345} 3 \mathrm{D}$ models from raw, noisy depth data captured from a depth ${ }_{346}$ sensor. To do so, for each voxel a $3 \mathrm{D}$ grid stores the signed 347 distance to the closest surface and a weight that indicates the 348 uncertainty of the surface measurement. This volumetric rep349 resentation and integration is based on the VRIP algorithm 350 [47]. One extracts the implicit surface of this representation 351 (i.e., reference model) by detecting zero-crossings (i.e., posi352 tions at which the distance sign changes) on the grid through ${ }_{353}$ a ray caster. This volumetric representation of KinectFusion is ${ }_{354}$ especially useful for the $\mathrm{F}+\mathrm{C}$ visualization based on the visible 355 background on MRI data technique, where the ray casting algo356 rithm is used to clip the 3D reference model directly from the ${ }_{357} 3 \mathrm{D}$ grid. Moreover, to keep the pyramid framework consistent, 358 a pyramid version of the ray cast data is built for each frame.

As evaluated in [48], the KinectFusion algorithm has max360 imum accuracy of approximately $10 \mathrm{~mm}$; therefore, it is as${ }_{361}$ sumed that its reconstructed models are suitable to be used as 362 reference for tracking and virtual data positioning in MAR ap363 plications which do not demand high accuracy. All of the steps 364 described above run in the GPU and are optimized for real-time 365 performance.

The 3D reference model is reconstructed only once, and it 367 is the basis for MAR live tracking. To position the medical 
368 volume into the scene, a semiautomatic registration method is 421 369 used [17]. The virtual data are coarsely aligned with the 3D ref370 erence model (which represents the patient's ROI data) in terms 371 of scale, positioning, and orientation. By controlling parame372 ters such as the scale factor, rotation angles, and translation vec373 tor, the user is able to make fine adjustments (e.g., rescale the 374 virtual data, change the position of the virtual data, or modify 375 the orientation of the virtual data) over the coarse registration 376 in order to produce a more visually pleasant integration of the 377 medical data into the augmented scene.

378 After the placement of the medical data into the scene, the 379 markerless tracking is started. In fact, live tracking is done 380 in two steps: during the reconstruction of the 3D reference 381 model, to align the different viewpoints acquired from the pa382 tient's ROI, and during the MAR with the patient and the med$38 з$ ical data. A real-time variant of the Iterative closest point (ICP) 384 algorithm [49] implemented on the GPU is used to estimate 385 the rigid transformation that aligns the current depth frame cap386 tured by the depth sensor with the previous one represented by ${ }_{387}$ the 3D reference model. To improve tracking accuracy without 388 too much impact on performance, we use a hierarchical vari389 ant of the ICP algorithm, similarly as done in [50]. Hence, 390 we estimate the camera pose starting from the coarsest level to 391 the finest one using the previously computed vertex and normal 392 map pyramid. After each iteration, we update the final cam${ }_{393}$ era pose estimated for the current frame. As discussed in the 394 Section 5, by controlling the number of iterations used for each 395 level of the tracking algorithm, we can trade off accuracy and 396 performance of the tracking in the MAR environment.

${ }_{397}$ As stated in $[46,50]$, the use of the 3D reference model for 398 tracking allows a more consistent rigid registration with less in399 cremental error. However, in the presence of fast rigid motion 400 between frames, the ICP algorithm may fail (i.e., not converge 401 to a valid result). Taking advantage of the fact that the main ${ }_{402} \mathrm{ROI}$ in this article is a head, we used a real-time head pose esti403 mation [51] to provide a new initial guess to the ICP algorithm 404 to compute correctly the current transformation [52].

\section{4. On-patient medical data visualization based on volume 406 clipping}

\section{4.1. Volume rendering}

408 Volume rendering is a field concerned with techniques for 409 synthesizing images from 3D scalar data. This problem of im410 age synthesis is mathematically formulated as a volume render${ }_{411}$ ing integral most commonly based on an emission-absorption 412 optical model [53].

${ }_{413}$ To synthesize the medical image, a single rendering pass ray 414 casting is applied over the bounding box of the medical volume 415 [54]. To improve image quality and performance of the volume 416 rendering, several techniques are used as follows:

- Stochastic jittering (i.e., random ray-start)—-to reduce sampling artifacts;

- Fast GPU-Based tricubic filtering-to reduce filtering artifacts $[55,56]$;
- Empty-space leaping—-to skip nonvisible voxels [57];

- Early ray termination-if the opacity accumulated is sufficiently high;

- Preintegrated transfer functions-to capture high frequencies introduced in the transfer functions defined with low sampling rates [58];

- Blinn-Phong shading with on-the-fly gradient computation-to add realism in the final rendering [59];

- GPU tricubic prefilter-to improve tricubic filtering accuracy [60];

- Volume clipping-to extract and emphasize importants parts of the volume [53].

434 In this work, the volume is clipped according to six planes ${ }_{435}$ parallel to the faces of the volume boundind box, although there ${ }_{436}$ are several alternative techniques for volume clipping, such as ${ }_{437}$ that in [61]. Nevertheless, we emphasize that the $\mathrm{F}+\mathrm{C}$ tech${ }_{438}$ niques can be used regardless of the technique used to crop the 439 volume.

440 After the volume rendering, medical data must be visualized ${ }_{441}$ in the augmented scene. To achieve this goal, $\mathrm{F}+\mathrm{C}$ visualization ${ }_{442}$ is used to show the medical data in a focus region in the context ${ }_{443}$ of the patient's body, as described in the next subsection.

\section{4.2. Focus and context visualization}

445 We present improvements over the $\mathrm{F}+\mathrm{C}$ visualization based ${ }_{446}$ on volume clipping proposed in [5]. When one is clipping a ${ }_{447}$ volume and rendering its image in an AR environment, there 448 will not be occlusion between the internal region of the volume 449 and the patient's ROI, as shown in left image in Figure 2.
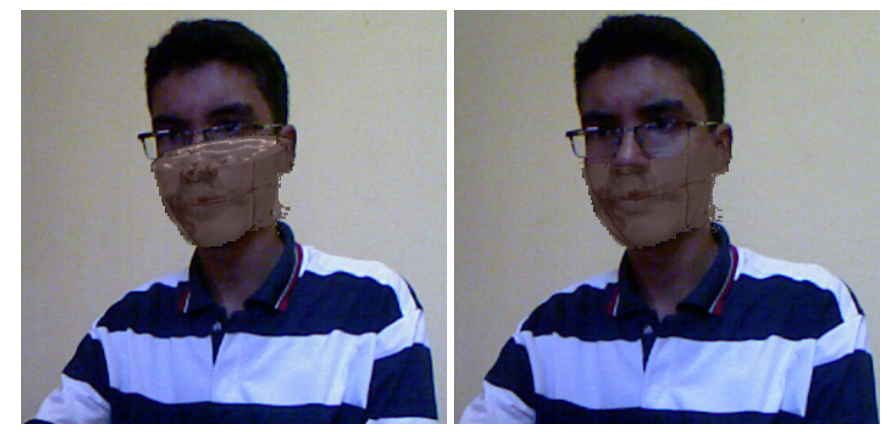

Figure 2: Occlusion between the volume's internal structures and the patient's region of interest. Left image: direct volume rendering with clipping. Right image: volume clipped rendered according to the proposed algorithm.

${ }_{450}$ If desirable, one can solve this issue by changing the single${ }^{451}$ pass ray casting [53]. We check if the first hit position of the ${ }_{452}$ ray cast in the volume is in the clipped region. If it is, the ray ${ }_{453}$ stops its traversal and is discarded from rendering. Otherwise, 454 the ray continues its traversal in the volume as normally done 455 in the standard ray casting algorithm. The visual effect of this 
456 algorithm can be seen in the right image in Figure 2, where 457 the internal structures of the volume were removed in the final 458 rendering.

\section{4.2.1. Smooth contours}

460 When a volume is clipped, to reveal hidden structures of the 461 medical data, and its image is rendered in an AR environment, 462 edges located at the intersection between the volume and the 463 clipping planes become visible. This visibility of the edges oc464 curs not only in this region, but also for the entire contour of the 465 volume rendered (Figure 3, left image).

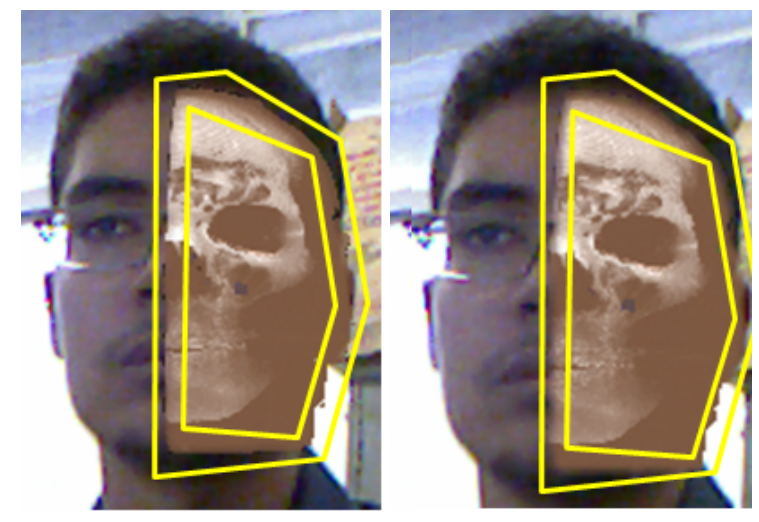

Figure 3: Influence of the smooth contours technique in the final rendering. Left image: direct volume rendering with clipping. Right image: volume clipped rendered according to the proposed algorithm. Contours are localized between the yellow shapes.

466 According to the $\mathrm{F}+\mathrm{C}$ technique presented in [2], one can 467 improve depth perception by smoothing the transition between ${ }_{468}$ the volume in the focus region and the rest of the AR scene. On 469 the basis of this, Macedo and Apolinario [5] proposed a new 470 method for $\mathrm{F}+\mathrm{C}$ visualization based on the smooth contours 471 technique, an algorithm that adds a smooth transition between 472 the volume rendered and the real scene based on the volume 473 contours.

${ }_{474}$ The smooth contours technique proposed in [5] consists of 475 the following steps: from the medical volume image, which is 476 loaded from the GPU to the CPU, it is converted to grayscale, 477 binarized by use of the threshold computed from Otsu's method 478 [62], contours are extracted from the method proposed by ${ }_{479}$ Suzuki [63] and are smoothed by use of a Gaussian blur (kernel ${ }_{480}$ size $3 \times 3$ pixels). The resulting image is a mask ( $\left.\alpha_{\text {smoothCont }}\right)$ 481 which weights the blending of the volume and the patient's 482 color images. Also, a factor $w_{c}$ can be dynamically defined 483 by the user to adjust the level of smoothing of the contours, ex${ }_{484}$ panding or compressing the area of operation of the algorithm ${ }_{485}$ (Equation 1). It ranges from 0, where the contours are ren486 dered, to $+\infty$, where the contour area is expanded, contours are ${ }_{487}$ smoothed, and then suppressed in the final rendering because to 488 the high level of smoothness required.

489 Instead of the technique running entirely on the CPU, we 490 propose an alternative method for the technique to run entirely 491 on the shader, improving performance and achieving the same
492 quality of the final rendering. To achieve this goal, the pipeline 493 is changed as follows (Figure 4, top part): the medical volume 494 image is binarized by use of a predefined threshold $t_{b}$, which op495 erates on the gray intensity of each pixel (empirically we have 496 found $t_{b}=0.1$ a good threshold for such a task), and the binary 497 image is blurred by one iteration of a two-pass Gaussian blur 498 (kernel size $3 \times 3$ pixels). Instead of explicitly computing the 499 contours by using Suzuki's method, we just apply the Gaussian 500 blur directly over the binary image. In practice, we have not 501 found a perceptual difference between these two approaches. 502 Moreover, as discussed in Section 5, with our new algorithm 503 we improved the performance of the original approach, as we 504 remove the need to transfer data from the GPU to the CPU, 505 which is a time-consuming step. Furthermore, because of the 506 separability of the Gaussian functions, the use of a two-pass 507 approach to convolve the binary image reduces the processing 508 time required by the filter while maintaining the same visual 509 result.

${ }_{510}$ As can be seen in Figure 3, the smooth contours technique ${ }_{511}$ softens the transition between the medical volume image and 512 the real scene. Furthermore, this method can be easily inte513 grated with other existing solutions, such as the CAM technique 514 [2]. An example of the result of such integration can be seen in ${ }_{515}$ Figure 5. In the top image in Figure 5, a circular mask is defined 516 over the window to select which parts of the medical volume 517 must be rendered into the augmented scene. With the CAM 518 method, there is no clear handling of the contours which result 519 from the clipping the volume. By using the smooth contours 520 technique (Figure 5, bottom image), we can solve this problem 521 by smoothing the contours inside the focus window.

${ }_{522}$ Two methods for $\mathrm{F}+\mathrm{C}$ visualization that take advantage of 523 the clipping effect and the concept of a visible background were ${ }_{524}$ proposed. They take advantage of the type of scanning technol525 ogy (CT or MRI) to enable new ways for physicians to visualize 526 and explore the medical data on the patient.

\section{4.2.2. Visible background on CT data}

${ }_{528}$ In volume rendering, CT data can be used to enable the vi${ }_{529}$ sualization of internal structures of the patient such as bones. ${ }_{530}$ By designing an appropriate transfer function, one can visual${ }_{531}$ ize the bone apart from the soft tissue of the volume. On the ${ }_{532}$ basis of the color values associated with the soft tissue, the vir${ }_{533}$ tual background used for rendering can be seen. In this case, it 534 is desirable to replace this virtual background by the real one, ${ }_{535}$ enhancing the visual perception of the scene. Moreover, by use 536 of this strategy, the visualization of the soft tissue is deempha${ }_{537}$ sized in the final rendering, emphasizing rather the focus region 538 of the visualization, the bone structure. The $\mathrm{F}+\mathrm{C}$ visualization 539 based on the visible background on CT data technique can be 540 applied to enable this kind of visualization. An overview of this ${ }_{541}$ method can be seen in the middle part of Figure 4.

${ }_{542}$ The background scene is captured and stored in memory. ${ }_{543} \mathrm{Next}$, the image of the volume after clipping is binarized and 544 sent to the shader as a foreground subtraction mask $I_{\text {subtraction }}$. ${ }_{545}$ This mask identifies the region where the background can be vi546 sualized on the basis of the gray intensity of the volume. Then, ${ }_{547}$ a user-defined threshold $w_{\text {grayLevel }}$ operates on the gray level of 


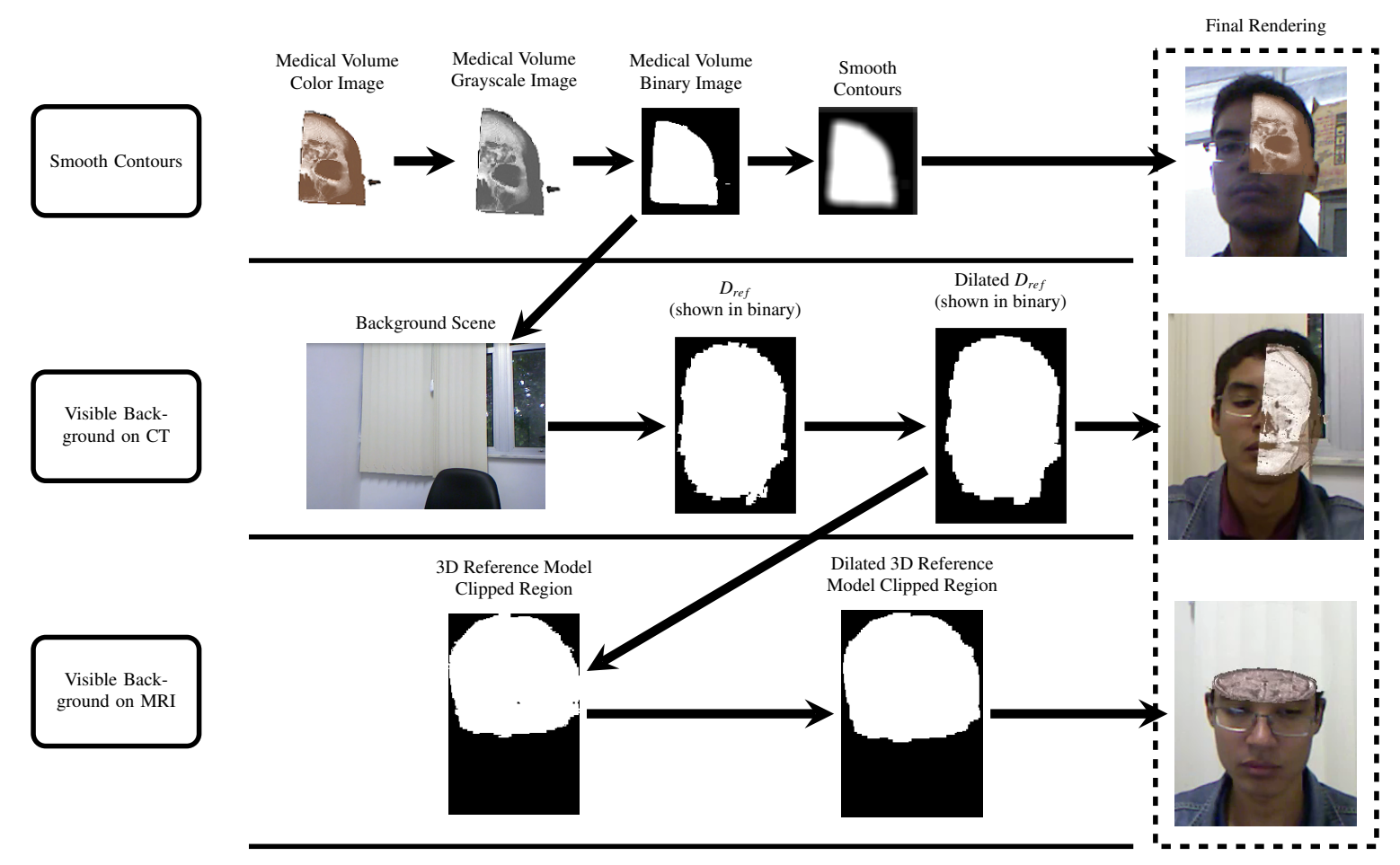

Figure 4: A schematic view of the proposed techniques. Focus plus context $(\mathrm{F}+\mathrm{C})$ visualization based on the smooth contours technique (top part): the blurred version of the binary image of the medical volume is used as a mask that smooths the transition between the medical data and the real scene on the final rendering. $\mathrm{F}+\mathrm{C}$ visualization based on visible background on $\mathrm{CT}$ data technique (middle part): from the binary image of the medical volume, the dilated image of the $3 \mathrm{D}$ reference model, and the background scene, the soft tissue of the medical data can be displayed merged with the background, emphasizing the visualization of the bone structure. $\mathrm{F}+\mathrm{C}$ visualization based on the visible background on MRI data technique (bottom part): by rendering a clipped image of the $3 \mathrm{D}$ reference model, the organs of the medical data can be displayed in the context of the patient's region of interest.

${ }_{548}$ the volume and separates bone and soft tissue regions, indicat549 ing where the background scene must be rendered.

${ }_{550}$ In our case, $D_{\text {ref }}$, the depth map of the $3 \mathrm{D}$ reference model, 551 does not overlap perfectly with the patient's ROI. To avoid the 552 presence of artifacts in the final rendering, $D_{\text {ref }}$ is dilated only 553 on its contours to preserve the original depth (which is used for 554 occlusion computation) and sent to the shader to represent the ${ }_{555}$ patient's ROI.

\section{4.2.3. Visible background on MRI data}

${ }_{557}$ In volume rendering, MRI data can be used to enable the 558 visualization of internal structures of the patient's anatomy such 559 as organs. In an AR environment, the best way to visualize 560 data of this kind is by clipping not only the medical volume but ${ }_{561}$ also the corresponding region of the patient's color image. In 562 this scenario, it is desirable to see the background scene in the 563 region clipped, which is the main goal of the visible background 564 on MRI data technique. An overview of this technique is given 565 in the bottom part of Figure 4.

566 The technique originally proposed in [5] is similar to the one 567 used for CT data. The background scene is saved. Next, tak568 ing advantage of the volumetric representation of KinectFusion, 569 which stores the 3D reference model as an implicit surface in 570 a 3D grid, one can clip the patient's ROI in real time. The 571 algorithm to render an image from the 3D clipped reference 572 model is given in Algorithm 1. This algorithm is an exten${ }_{573}$ sion of the pseudocode presented in [46]. We ray-cast the $3 \mathrm{D}$
574 grid, and when the ray traverses a zero-crossing position (i.e., 575 the silhouette of the 3D reference model stored in the volume) 576 and it is in the clipped region, the voxel's corresponding pixel 577 is rendered in the output image. The medical volume is clipped 578 separately and sent to the shader. The output image from this 579 algorithm is $I_{\text {subtraction, }}$, which is used with the same objective 580 as described for the visible background on CT data technique. ${ }_{581}$ Both $I_{\text {subtraction }}$ and $D_{\text {ref }}$ are dilated because of the problem of 582 overlapping described before.

${ }_{583}$ The algorithm proposed in [5] is subject to the presence of 584 artifacts at the intersection between the clipping plane and the ${ }_{585} 3 \mathrm{D}$ reference model. To mitigate their effects, we use adap${ }_{586}$ tive sampling to reduce the step size of the ray when it is near 587 the zero-crossing position. We check this proximity by using 588 a specific threshold $\left(t_{\text {prox }}\right)$ over the truncated signed distance 589 function stored at the voxel $g$ being traversed $\left(g_{t s d f}\right)$. When 590 near the zero-crossing, the step size of the ray cast is reduced to 591 the value $w_{s}$ to perform a more accurate traversal. From empir592 ical tests, we have set $t_{\text {prox }}=0.5$ and $w_{s}$ equals to one fourth of 593 the original step size. As shown in Section 5, by using this new 594 algorithm, we improved the visual quality of the method while 595 maintaining almost the same performance.

\section{4.3. Final rendering}

After the volume rendering, the color frame buffer is sent 598 to the shader for blending with the patient's color data coming 599 from the RGB-D sensor. For the CAM and smooth contours 


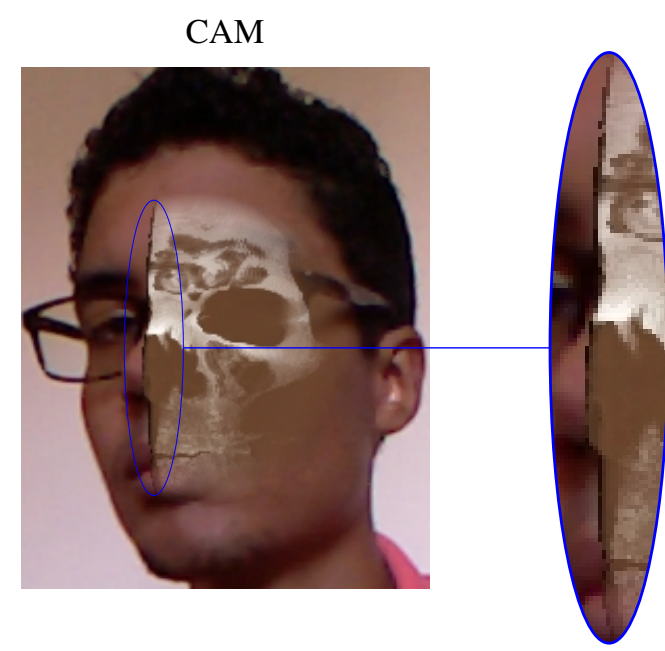

$\mathrm{CAM}+$ Smooth Contours

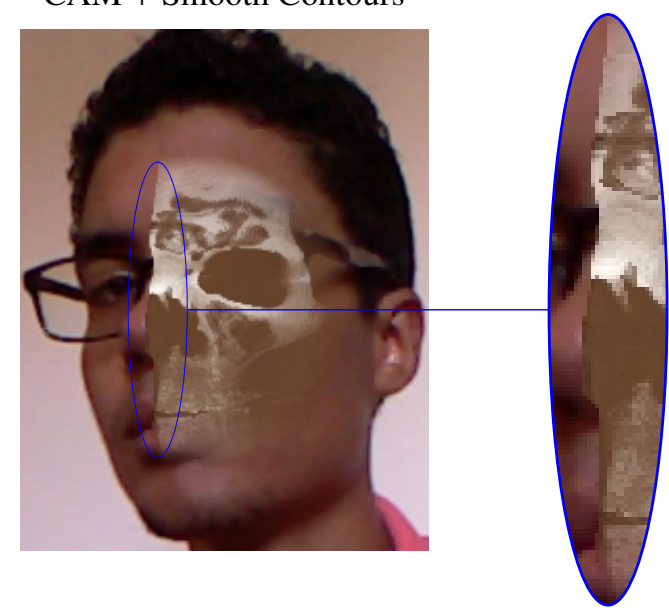

Figure 5: Focus plus context visualization based on the contextual anatomic mimesis (CAM) algorithm (top image) and its extension with the smooth contours technique (bottom image). By using the smooth contours technique, we can suppress the black border resulting from the clipping of the medical data, allowing a more seamless transition between real and virtual images.

600 techniques, blending is done by the following linear interpola601 tion:

602

$$
I_{\text {final }}=\beta I_{\text {real }}+(1-\beta) I_{\text {medical }}
$$

603 where $I_{\text {real }}$ is the image captured by the sensor, $I_{\text {medical }}$ is the 604 image corresponding to the medical volume, and $I_{\text {final }}$ is the 605 resulting augmented image. In our approach, $\beta$ is defined dy606 namically, for every fragment/pixel, by the $\mathrm{F}+\mathrm{C}$ visualization 607 techniques mentioned before, according to the following equa608 tion:

$$
\beta=\operatorname{clamp}\left(\max \left(w_{c}\left(1.0-\alpha_{\text {smoothCont }}\right), \alpha_{C A M}\right)\right)
$$

610 where clamp is a function that clamps the input parameter to 611 the interval $[0,1]$.

612 For the visible background-based $\mathrm{F}+\mathrm{C}$ techniques, the ${ }_{613}$ shader listed in Algorithm 2 is used instead of Equation 1, be${ }_{614}$ cause this Equation does not include the background rendering.

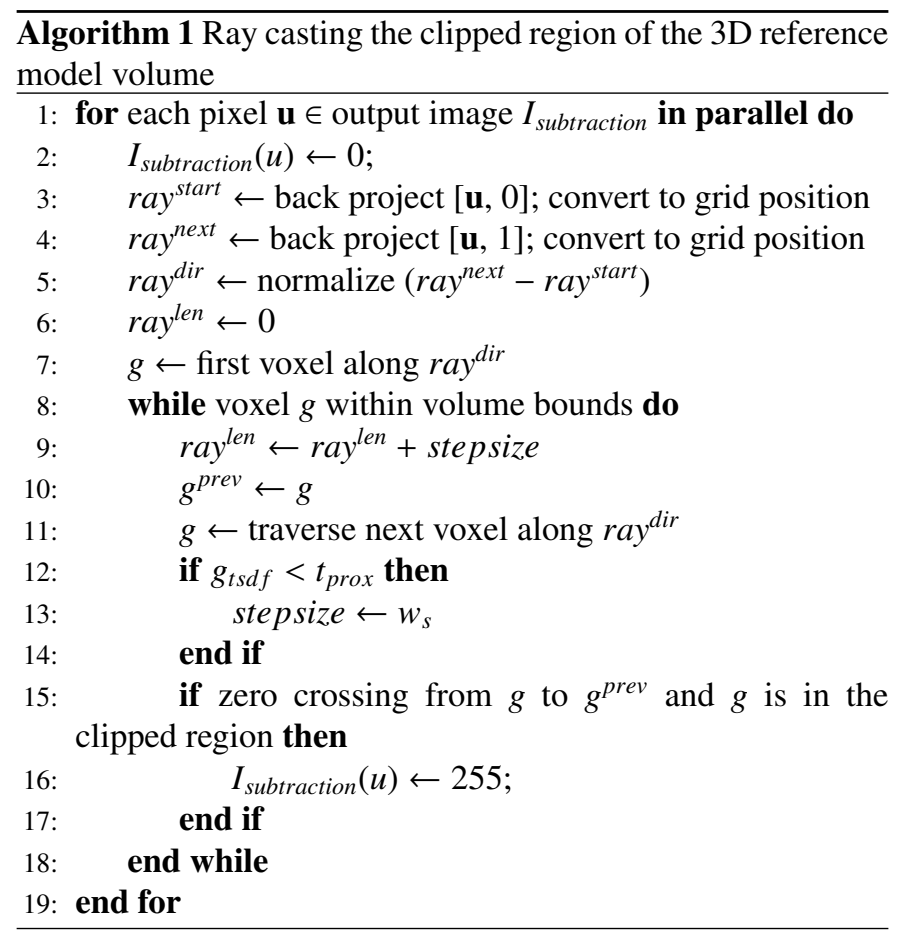

615 The algorithm for the visible background on CT data tech616 nique can be seen in lines 1-15 and 22-24. The color image 617 captured from the RGB-D sensor is rendered in the region that ${ }_{618}$ does not represent the patient's ROI (i.e., where the depth of the ${ }_{619} 3 \mathrm{D}$ reference object is zero, as it was not reconstructed) (lines $6202-4)$. The captured color image is also rendered when the vol621 ume is occluded and the occludee has depth (i.e., it is not in 622 a hole region) (lines 5-7). Next, if the fragment is in the sub623 traction mask region, the volume or the background scene is 624 rendered. Otherwise, the fragment is in the clipped region and 625 the real color image is rendered (lines 23-24). Gray intensity is 626 computed from the volume (by the gray function) and assigned 627 to $\beta$. Considering that the bone is rendered with a gray level ${ }_{628}$ greater than the soft tissue's and than $w_{\text {grayLevel }}$, it is rendered 629 without the background scene. Assuming that bone and soft ${ }_{630}$ tissue have different gray intensities, $w_{\text {grayLevel }}$ can be adjusted ${ }_{631}$ to render the bone with its original color and the soft tissue can 632 be linearly interpolated with the background scene (lines 8-15). ${ }_{633}$ The algorithm for the visible background on MRI data tech634 nique is shown in lines 1-8 and 16-24. The color image cap635 tured from the RGB-D sensor is rendered in the same way as ${ }_{636}$ described for the visible background on CT data technique. The 637 main difference here is that if the subtraction mask is active (i.e., 638 the patient's ROI is clipped) and if there are medical data to be 639 visualized, they are rendered. Otherwise, the background im640 age is rendered.

${ }_{641}$ In an AR environment, it is desirable to solve the problem 642 of occlusion between virtual and real data. For a specific view${ }_{643}$ point, depth images of the patient's 3D reference model $D_{\text {ref }}$ ${ }_{644}$ and the 3D object coming from the sensor's live stream $D_{\text {live }}$ 645 are used to solve this issue. If the depth from $D_{\text {live }}$ is lower ${ }_{646}$ than that from $D_{\text {ref }}$, the object captured by the sensor is in front 647 of the reference object and the medical volume is the ocludee, 
${ }_{648}$ otherwise, the medical volume is the occluder.

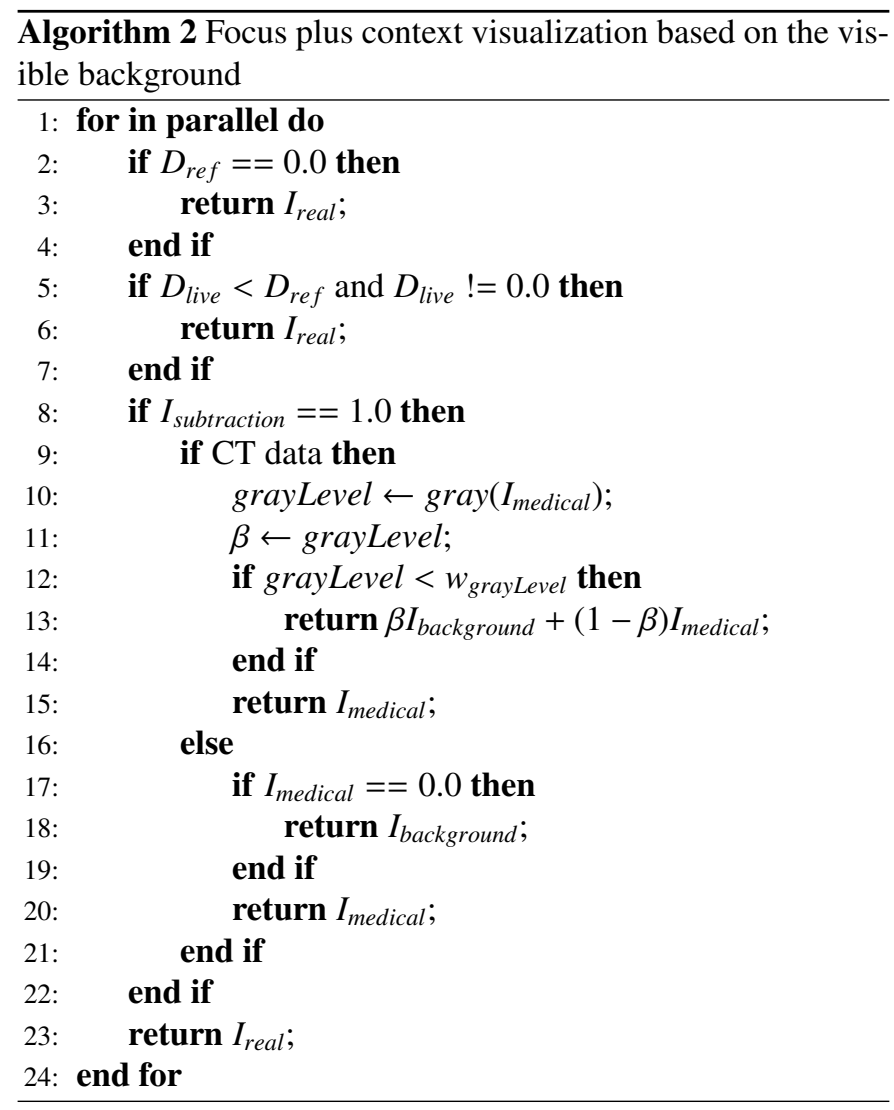

\section{${ }_{649}$ 5. Experimental results}

650 In this section, the performance and visual quality of the $\mathrm{F}+\mathrm{C}$ 651 visualization techniques based on volume clipping are evalu652 ated.

\section{5.1. Experimental setup}

654 For all tests, as the computer we used an Intel Core ${ }^{\mathrm{TM}}$ i7$6553770 \mathrm{~K}$ CPU (3.50 GHz), 8GB RAM, and a NVIDIA GeForce ${ }_{656}$ GTX 660 graphics card. For 3D reference model reconstruc657 tion, we used the open-source $\mathrm{C}++$ implementation of Kinect${ }_{658}$ Fusion released by the Point Cloud Library project [64].

659 We use a Microsoft Kinect device as a low-cost, accessible, 660 and versatile RGB-D sensor [65]. The medical dataset used ${ }_{661}$ was a CT volumetric dataset of a head released by the Visible ${ }_{662}$ Human Project [66] of resolution $128 \times 256 \times 256$, an MRI vol663 umetric dataset of a head from MRI Head available in Volume ${ }_{664}$ Library [67] of resolution $256^{3}$, and an MRI dataset of a knee 665 of resolution $400 \times 400 \times 250$ and a CT dataset of a torso of res${ }_{666}$ olution $512 \times 512 \times 288$, both available in OsiriX [68]. The 3D 667 reference models were reconstructed with KinectFusion with a 668 grid with resolution of $512^{3}$.

\section{5.2. Performance evaluation}

670 In our preprocessing computation, the 3D reference model 671 was reconstructed at 40 FPS. From empirical tests, the user
672 takes less than $10 \mathrm{~s}$ to place the volume into the scene and ad673 just it. The markerless live tracking and volume rendering tech674 niques together run at 45 FPS. These performance results are 675 the same as those reported in previous work [5]; however, they 676 were computed without taking into consideration the depth sen677 sor's performance ${ }^{1}$.

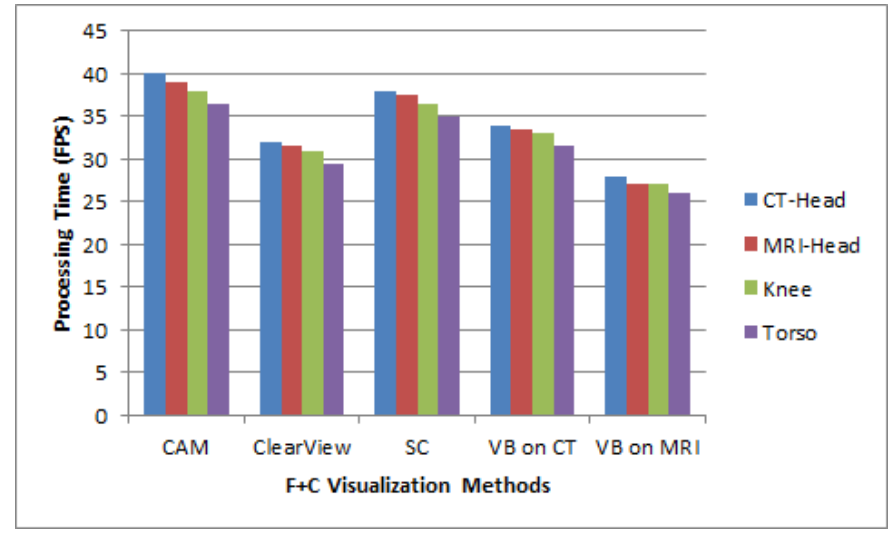

Figure 6: Performance results measured in frames per second (FPS) for each one of the focus plus context $(\mathrm{F}+\mathrm{C})$ visualization techniques discussed in this article. CAM, contextual anatomic mimesis; SC - smooth contours; VB - visible background.

678 The performance of the on-patient medical data visualization 679 for the $\mathrm{F}+\mathrm{C}$ visualization techniques used in this work can be 680 seen in Figure 6. The performance was evaluated for all four 681 medical datasets described in Section 5.1.

682 From Figure 6, we see that the CAM technique provides the ${ }_{683}$ best performance, which is expected since this technique does 684 small computations directly on the shader. For ClearView, an ${ }_{685} \mathrm{~F}+\mathrm{C}$ visualization technique proposed specifically for volume 686 rendering [30], we generated only one context layer and one 687 focus layer in our application. In the context layer, we com688 puted three layers (i.e., position, normal and curvature) for the 689 medical volume. For the focus layer, we rendered an isosurface 690 from the medical volume, according to a user-defined iso-value. 691 The layers were recalculated for every change in viewpoint. In 692 our application, ClearView requires approximately $6.25 \mathrm{~ms}$ to 693 compute and render these layers, which are composed accord694 ing to the distance-based importance shader [30], decreasing 695 the application's performance to a frame rate even lower than 696 that provided by the smooth contours and visible background 697 on CT data techniques. For the smooth contours technique, by 698 transferring all the pipeline to the GPU, we obtained a huge im699 provement over the original technique proposed in [5], which 700 achieved 20 FPS (for CT-Head in Figure 6) on the same hard701 ware. The visible background on CT data technique runs in full 702 real time because it operates mostly on the shader. Dilation ap${ }_{703}$ plied on $D_{\text {ref }}$ decreases the application's performance slightly. 704 The visible background on MRI data technique is slower than 705 the other techniques because of the ray casting performed on 706 the 3D reference model to render the clipped patient's ROI.

\footnotetext{
${ }^{1}$ The Kinect sensor acquires depth data at 30 FPS; hence, this limits the maximum performance of the application.
} 
707 However, differently from [5], we added an adaptive sampling 708 scheme to improve the visual quality of the approach. This 709 adaptive approach allowed us to obtain the same performance 710 as the original technique. Moreover, all of the techniques run at 711 more than 25 FPS, therefore in real time, even for the medical 712 dataset of highest resolution (Torso in Figure 6).

\section{5.3. Visual quality evaluation}

714 By the use of the shader proposed in Algorithm 2, occlusion 715 is supported by our application, as can be seen in Figure 7.

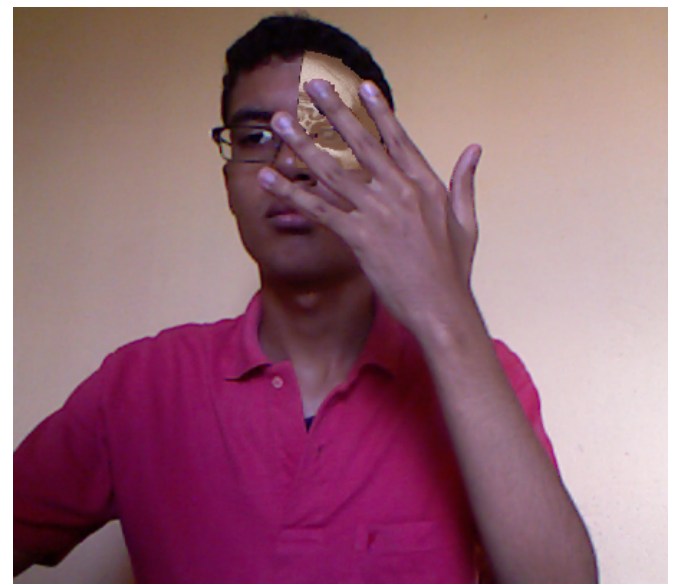

Figure 7: Occlusion support is achieved by comparing depth values from current and previous depth frames.

716 For the $\mathrm{F}+\mathrm{C}$ visualization based on the smooth contours tech717 nique, the level of smoothness can be controlled by the param718 eter $w_{c}$. As can be seen in Figure 8, the transition between the 719 volume and the real scene becomes smoother as $w_{c}$ increases. ${ }_{720}$ At the same time, the volume contours become less visible.
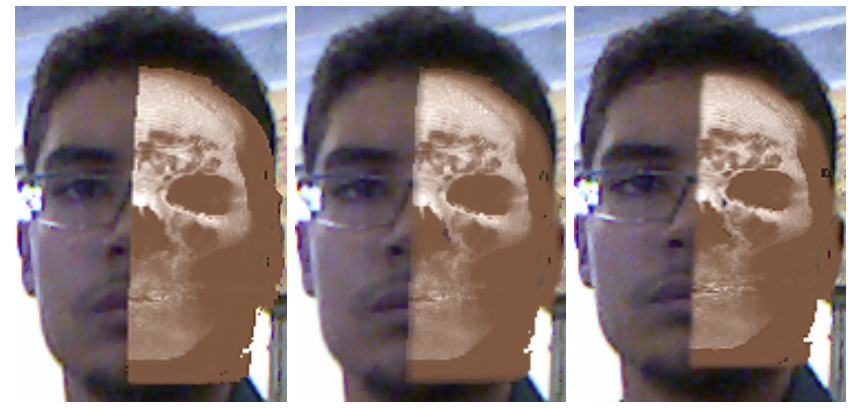

Figure 8: Influence of the parameter $w_{c}$ in the smooth contours technique. Left image: $w_{c}=0$. Middle image: $w_{c}=2$. Right image: $w_{c}=4$.

${ }_{721}$ For the $\mathrm{F}+\mathrm{C}$ visualization based on the visible background on ${ }_{722}$ CT data technique, bone and soft tissue structures can be sepa${ }_{723}$ rated with use of $w_{\text {grayLevel }}$. From Figure 9, it can be seen that 724 by changing this parameter, we can render the volume without 725 the background scene, with the soft tissue linearly interpolated 726 with the background scene or almost completely invisible.

${ }_{727}$ For the $\mathrm{F}+\mathrm{C}$ visualization based on the visible background on 728 MRI data technique, we have proposed an improvement to miti${ }_{729}$ gate artifacts resulting from clipping of the patient's ROI [5]. A
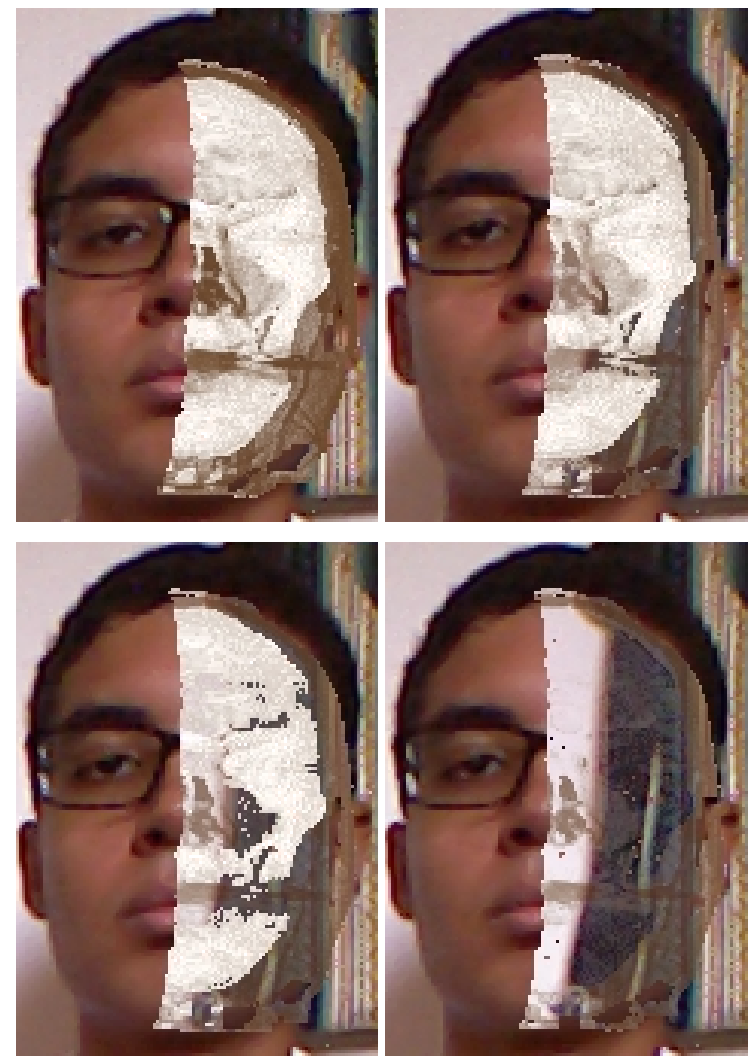

Figure 9: Influence of $w_{\text {grayLevel }}$ in the visible background on CT data technique. Top-left image: $w_{\text {grayLevel }}=0$. Top-right image $w_{\text {grayLevel }}=0.5$. Bottom-left image: $w_{\text {grayLevel }}=0.75$. Bottom-right image: $w_{\text {grayLevel }}=1$.

730 visual comparison between our approach and the one proposed ${ }_{731}$ in [5] can be seen in Figure 10. As the artifacts become more ${ }_{732}$ visible during the user's movement, this figure shows the pa${ }_{733}$ tient's ROI in different positions and the presence of artifacts in 734 these scenarios. Moreover, regions around the contours of the 735 clipped data are zoomed to enable a clear visualization of the ${ }_{736}$ problems of related work [5] in comparison with the improve${ }_{737}$ ments proposed here. Artifacts at the intersection between the ${ }_{738}$ patient's ROI and the clipping plane are more visible when the 739 user rotates his or her head in front of the sensor [5]. From col740 umn I iin Figure 10, we can see artifacts arising at the contours. ${ }_{741}$ By use of our approach (Figure 10, column II), artifacts are mit742 igated and the results are comparable to a scenario (Figure 10, 743 column III) where the ray is cast in a uniform sampling way and 744 the step size of the ray is too small to render the clipped data 745 in an AR application. In this case, our method has better per746 formance than the best visual quality scenario, as ours runs at 74728 FPS, whereas because of its use of ray casting with a small 748 step size, the ground-truth approach runs at only 9 FPS, which 749 does not provide performance that is enough for an interactive 750 application [69].

751 Our MAR environment supports not only rendering of the 752 head, but also rendering of other ROI in the patient's body. The 753 on-patient visualization of the torso and knee datasets with the ${ }_{754}$ CAM technique is shown in Figure 11, and can be found in the 

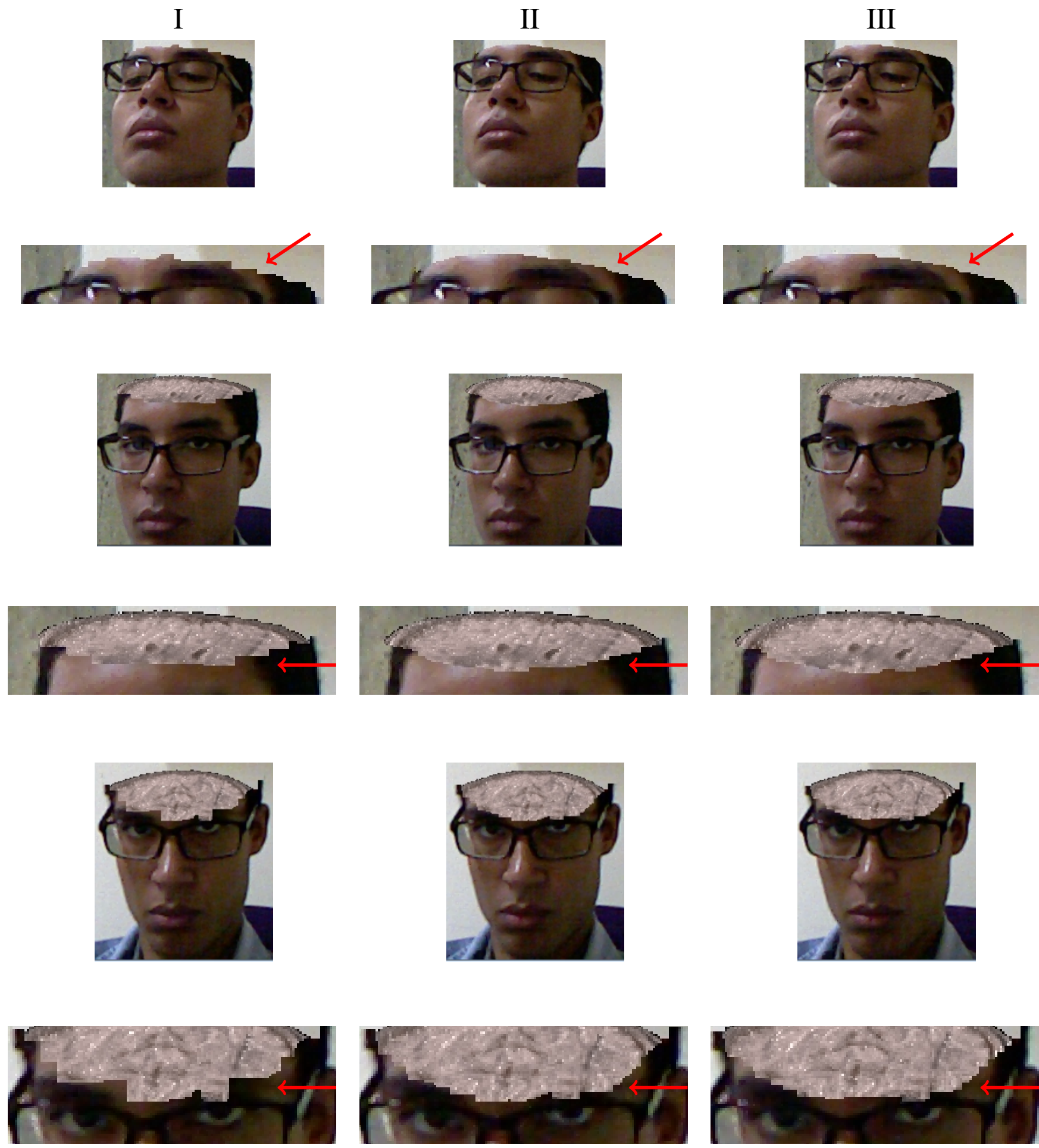

Figure 10: Different schemes for focus plus context visualization based on the visible background on MRI Data technique. Column I results from the application of the original technique proposed in [5], column II refers to the adaptive scheme proposed in this article, and column III represents a ground-truth scenario where the ray casting performs uniform sampling and the step size of the ray is too small to render the clipped data in an interactive application. Our adaptive approach (column II) is three times faster than the ground-truth scenario (column III), while achieving almost the same visual quality. For each image, we zoom in on the contours of the clipped data to highlight the differences between the different approaches. Furthermore, red arrows are used to show regions where the visual difference is apparent. The presence of alias in the zoomed images is due to the digital zoom.

755 supplementary video. Even with different ROI, our MAR envi- ${ }_{758}$ prises mainly the abdomen and the pelvis, we have found it 756 ronment tracks the 3D reference model and shows the medical 759 useful to show them separately.

${ }_{757}$ data at the position of the patient's anatomy. As the torso com-

760 The process to augment these structures over the patient's 
${ }_{761}$ body is almost the same as the one described in the previous ${ }_{762}$ sections. The small adaptations required to make the augmen763 tation of other structures possible are discussed in Section 6.
I
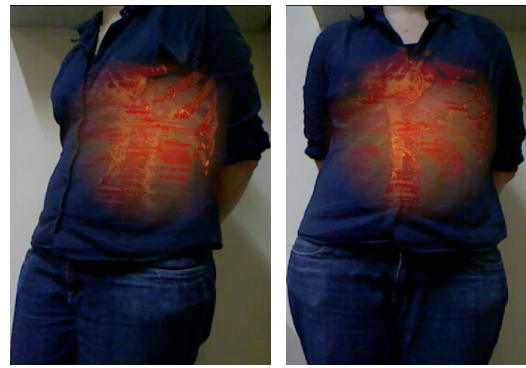

II
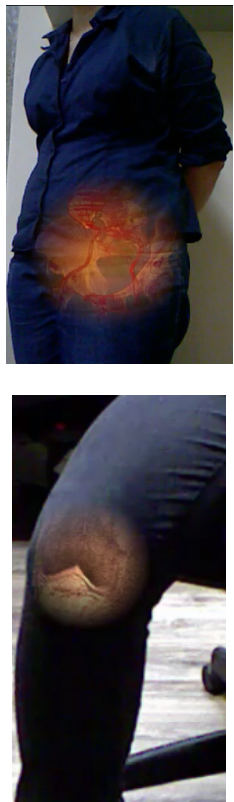
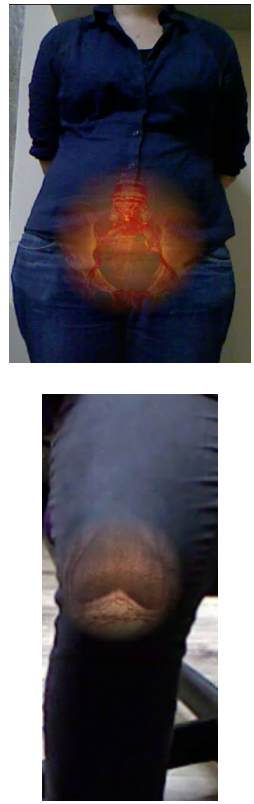
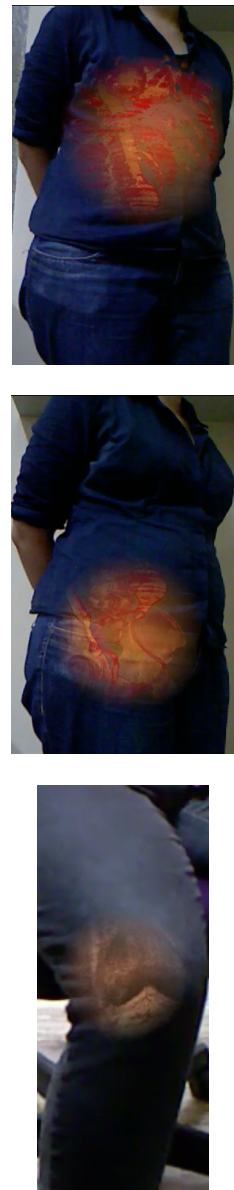

Figure 11: On-patient visualization of CT torso (rows I and II) and MRI knee (row III) datasets. To highlight the features of the CT torso dataset, we show the abdomen (row I) and pelvis (row II) separately.

64 Depending on the transfer function and/or the structure of the 765 medical dataset, the ray casting technique may render holes in 766 the final image (Figure 12, left image). In this case, the $\mathrm{F}+\mathrm{C}$ 767 techniques handle the holes in different ways, achieving dif768 ferent results for the final rendering. An example of this can be 769 seen in the right image in Figure 12, where the smooth contours 770 technique replaces the virtual background color of the hole by 771 the color captured by the RGB-D sensor and smooths the con772 tours around the hole region. The visible background on CT 773 data technique replaces the virtual background color of the hole 774 by the background color of the real scene, which was captured 775 previously.

\section{5.4. Accuracy evaluation}

777 In all the experiments, the patient's ROI is augmented with 778 a generic volumetric dataset. The use of a generic volume 779 does not affect our visual quality evaluation since the volume 780 is scaled and positioned semiautomatically with the user's fine 781 adjustments [17]. In this way, the accuracy of the registration
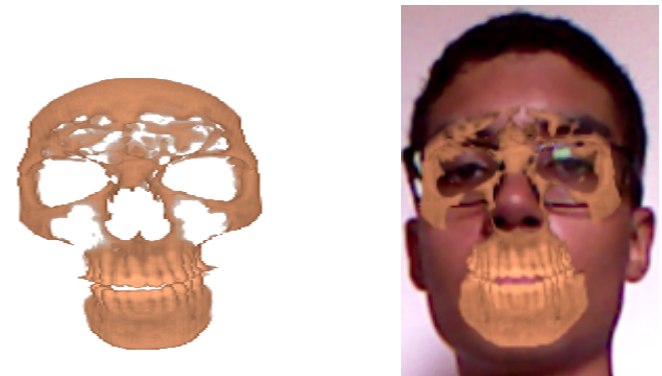

Figure 12: Medical volume with holes (left) rendered into the augmented scene with the smooth contours plus contextual anatomic mimesis (CAM) technique (right). For this figure, CAM's fall-off weight was set to 0 .

782 between the medical data and the patient's ROI depends on the ${ }_{783}$ quality of the user's fine adjustment because of the use of a ${ }_{784}$ generic volume.

${ }_{785}$ Related to the accuracy of the MAR environment, 3D recon786 struction has accuracy of approximately $10 \mathrm{~mm}$ [48], and by us787 ing the hierarchical ICP algorithm, we improved the live track788 ing accuracy from approximately $3 \mathrm{~mm}$ [5] to approximately $7892 \mathrm{~mm}$, according to the point-to-plane error metric [49]. In 790 this environment, tracking error does not accumulate between 791 frames.

\section{6. Discussion}

793 As mentioned in Section 1, inspired by the field of on-patient 794 craniofacial data visualization, we evaluated performance and 795 visual quality of the proposed techniques in a scenario where 796 the patient's ROI consists of the patient's head. In other con797 texts, where the ROI can be another part of the body, such as 798 the abdomen, pelvis and knee (Figure 11), one can still use the 799 solution presented in this article with minor adaptations. How800 ever, the MAR environment may still require some additional ${ }_{801}$ changes to reconstruct and track the poses. The main problems 802 related to these adaptations rely on the segmentation, tracking 803 and reconstruction of the patient's ROI.

804 To segment the patient's ROI in the scene, we propose the 805 use of a classification algorithm to detect and segment it from 806 the color image. This solution is desirable for a few ROI, such 807 as the head and hand. For others, which do not have classifica808 tion algorithms available to perform such a task, one solution 809 is to position the ROI relatively distant from the background 810 scene and segment it in the depth image with background seg811 mentation through $z$-axis thresholding. For the scenarios shown 812 in Figure 11, we used this strategy based on depth segmentation 813 to remove the background scene.

${ }_{814}$ Depth-based tracking algorithms (e.g., ICP) are dependent on 815 the presence of geometric information on the scene [70]. Some 816 ROI, such as the arm and leg, do not have much variation in 817 the depth values captured by the sensor between different view${ }_{818}$ points. In this case, a texture-based tracking algorithm which 819 operates according to the features of the color image [71, 72] 
${ }_{820}$ can be used to improve tracking accuracy. Furthermore, mark821 erless tracking uses geometric data of part of the real scene as 822 a natural marker. The natural marker (i.e., in our case the pa823 tient's ROI) may suffer nonrigid motion if it is a deformable 824 object. For the patient's face or hand, for instance, it is desir825 able for the tracking algorithm to support non-rigid interaction 826 between the patient and the application. Despite the complexity 827 of nonrigid registration, there are some methods which provide ${ }_{828}$ real-time performance $[73,74]$ and can be used together with 829 the markerless rigid tracking used in this work to improve ac830 curacy and robustness for tracking of deformable structures. In ${ }_{831}$ all tests reported in this article, we used only the ICP algorithm 832 for tracking.

${ }_{833}$ For the hand and foot, ROI which contain smaller struc834 tures (e.g. fingers), more accurate 3D reconstruction algorithms ${ }_{835}[75,73]$ may be required to reconstruct a $3 \mathrm{D}$ reference model 836 which captures the finest details of the patient's ROI, hence ${ }_{837}$ enabling high-quality tracking and occlusion handling even in ${ }_{838}$ these smaller structures. In this situation, the KinectFusion al${ }_{839}$ gorithm is able to reconstruct acceptable 3D reference mod840 els for such ROI, although showing some artifacts which can ${ }_{841}$ have some impact on tracking accuracy. In this work, we used 842 only the KinectFusion algorithm to reconstruct the 3D reference 843 models.

${ }_{844}$ As can be seen in the supplementary video, the use of a 3D 845 reference model as a basis for markerless registration allows 846 tracking of the medical data not only when the center of ro847 tation is located at the position of the patient's anatomy. For 848 knee visualization (Figure 11), the center of rotation is located 849 mainly in the torso of the user, which is translated in relation 850 to the knee region. Even in this case, our MAR environment ${ }_{851}$ supports the tracking of the medical data into the augmented 852 scene.

${ }_{853}$ By using the hierarchical tracking algorithm, we improved 854 tracking accuracy, as mentioned in Section 5. The advantage of 855 this improvement is twofold: for the reconstruction of the 3D ${ }_{856}$ reference model, in which the viewpoints captured by the depth ${ }_{857}$ sensor are rigidly aligned with more accuracy, resulting in a 858 more accurate 3D reference model reconstruction; for the AR 859 tracking, giving more tracking stability and less misalignment ${ }_{860}$ between real and virtual objects.

${ }_{861}$ As already known in the field of AR, tracking technologies 862 may suffer from jittering. As can be seen in the supplemen${ }_{863}$ tary video, even when we used the hierarchical ICP algorithm ${ }_{864}$ together with the head pose estimation solution to improve 865 tracking accuracy and robustness, the MAR environment is still 866 prone to jittering when the user moves his or her ROI in front 867 of the depth sensor. To minimize the jittering, one can increase 868 the number of ICP iterations to trade off tracking accuracy and ${ }_{869}$ performance or change the tracking algorithm for another one 870 which can explicitly handle such a problem. Hence, we em871 pirically have found it useful to use only three iterations of the 872 hierarchical ICP algorithm (i.e., one for each level of the pyra$873 \mathrm{mid}$ ), prioritizing performance over accuracy.

874 Markerless tracking solutions are not as accurate as some 875 commercial marker-based solutions. When developing a medi876 cal AR application, one must decide carefully which of these
877 technologies to use. Although being intrusive in the scene, 878 marker-based solutions may ease the positioning of the medi879 cal data into the scene and the tracking of the medical data with 880 high accuracy, being recommend for medical applications that 881 deal with surgery, as done in [2]. Markerless solutions are not 882 very accurate, and are therefore recommended for applications 883 which demand visually appealing results for the composition of 884 real and virtual data, typically medical applications developed 885 for training or visualization purposes [7].

886 For on-patient medical data visualization, the proposed ap${ }_{887}$ plication supports CT and MRI data, but can be easily extended 888 to support other scanning data as well. Medical data with res889 olution higher than $512^{3}$ can be used for a cadaver or phantom 890 study. For such scenarios, the only difference is that the ROI 891 is static in the scene and it is the sensor that must be moved to 892 capture different viewpoints and reconstruct a single $3 \mathrm{D}$ refer893 ence model. We have evaluated the $\mathrm{F}+\mathrm{C}$ techniques only for an 894 in-vivo study with different users as a patient.

895 In AR applications, one must pay attention to the way in 896 which the virtual content will be visualized in the augmented 897 scene. In this article, we have described three techniques to 898 improve the depth perception in medical AR scenarios. The ar899 tifacts present in the visible background on MRI data technique 900 proposed in [5] decrease the quality of the final image in the re901 gion of the clipped medical data. This problem is even severer 902 because of the high spatial and temporal discontinuity of the ar903 tifacts. Through the use of an adaptive scheme where the ray 904 casting samples more voxels only at the location of the clipping 905 plane, we achieved high-quality images (Figure 10, column II), 906 almost indistinguishable from the ground-truth images shown 907 in column III in Figure 10.

${ }_{908}$ The techniques for $\mathrm{F}+\mathrm{C}$ visualization based on the visible 909 background do not support the visualization of real dynamic 910 background scenes. In this case, we cannot use the color camera 911 of the RGB-D sensor because the patient occludes part of the 912 background being captured. A multiview approach, in which 913 an additional webcam is used to capture the real background 914 scene, may solve this problem.

\section{7. Conclusion and future Work}

We have presented improvements for on-patient medical data 917 visualization by using $\mathrm{F}+\mathrm{C}$ visualization and volume clipping. ${ }_{918}$ The performance and visual quality of the proposed techniques 919 were evaluated, and from the tests conducted, we conclude that 920 they are capable of running in real time and improve the visual ${ }_{921}$ quality of the final scene. To further enhance the quality of the 922 integration of the virtual data into the augmented scene, occlu${ }_{923}$ sion is handled and tracking accuracy is improved. Finally, we 924 have shown that our approach is versatile such that it can be ${ }_{925}$ used for different ROI of the patient.

926 In future work, we intend to evaluate the full solution (MAR 927 environment and $\mathrm{F}+\mathrm{C}$ techniques) in a real medical training en928 vironment, where high accuracy is not required for the applica929 tion. Further, an in-depth study must be conducted to improve 930 accuracy for scenarios where the medical dataset of the patient ${ }_{931}$ must be used for the on-patient visualization. 
${ }_{932}$ With feedback from specialists, we will be able to improve or ${ }_{993}$ ${ }_{933}$ adapt the methods where needed or even to collect a database of 9 ${ }_{934}$ craniofacial data to further improve future tests and evaluations 935 of our approach.

${ }_{936}$ For all the $\mathrm{F}+\mathrm{C}$ visualization techniques proposed in this ar${ }_{937}$ ticle, quantitative evaluation and extensive user study must be ${ }_{938}$ conducted to validate the proposed techniques from the per939 spective of the final users.

${ }_{940}$ For the AR environment, we used a conventional display to 1 ${ }_{941}$ show the augmented scene. Multiview solutions based on AR 942 glasses or portable solutions based on mobile devices can be ${ }_{943}$ used, where the proposed approach is performed on a server 944 and the visualization of the augmented content is transferred to 945 those alternative hardware devices, allowing a seamless visual946 ization of the virtual content on the real scene.

${ }_{947}$ The markerless tracking algorithm fails if the patient's ROI 948 is not visible in the view of the RGB-D sensor and the algo949 rithm does not support relocalization nor nonrigid registration 950 of the 3D reference model. These features must be supported 951 to further enhance the accuracy and robustness of the tracking.

\section{Acknowledgments}

953 We are grateful to the Point Cloud Library project for provid954 ing the open-source implementation of the KinectFusion algo${ }_{955}$ rithm. We are also grateful to Gabriele Fanelli for providing the 956 open-source implementation of her real-time head pose estima957 tion algorithm. We thank Rafaela Souza for being the model in 958 some experiments of this study. This research is financially sup959 ported by Fundação de Amparo à Pesquisa do Estado da Bahia 960 (FAPESB) and Coordenação de Aperfeiçoamento de Pessoal de ${ }_{961}$ Nível Superior (CAPES).

[1] Azuma R, Baillot Y, Behringer R, Feiner S, Julier S, MacIntyre B. Recent advances in augmented reality. IEEE Comput Graph Appl 2001;21(6):34-47. doi:10.1109/38.963459.

[2] Bichlmeier C, Wimmer F, Heining SM, Navab N. Contextual anatomic mimesis hybrid in-situ visualization method for improving multi-sensory depth perception in medical augmented reality. ISMAR '07; Washington, DC, USA: IEEE. ISBN 978-1-4244-1749-0; 2007, p. 1-10.

[3] Kutter O, Aichert A, Bichlmeier C, Traub J, Heining SM, Ockert B et al. Real-time Volume Rendering for High Quality Visualization in Augmented Reality. In: AMI-ARCS 2008. New York, USA: MICCAI Society; 2008,.

[4] Wieczorek M, Aichert A, Kutter O, Bichlmeier C, Landes J, Heining SM, et al. GPU-accelerated Rendering for Medical Augmented Reality in Minimally-Invasive Procedures. In: BVM 2010. Springer; 2010,

[5] Macedo MCdF, Apolinario AL. Improving on-patient medical data visualization in a markerless augmented reality environment by volume clipping. In: Graphics, Patterns and Images (SIBGRAPI), 2014 27th SIBGRAPI Conference on. 2014, p. 149-56. doi:10.1109/SIBGRAPI.2014.33.

[6] Card SK, Mackinlay JD, Shneiderman B, editors. Readings in Information Visualization: Using Vision to Think. San Francisco, CA, USA Morgan Kaufmann Publishers Inc.; 1999. ISBN 1-55860-533-9.

[7] Debarba HG, Grandi J, Maciel A, Zanchet D. Anatomic hepatectomy planning through mobile display visualization and interaction. In: MMVR; vol. 173. IOS Press. ISBN 978-1-61499-022-2; 2012, p. 111-5.

[8] Kato H, Billinghurst M. Marker tracking and hmd calibration for a videobased augmented reality conferencing system. In: Augmented Reality, 1999. (IWAR '99) Proceedings. 2nd IEEE and ACM International Workshop on. 1999, p. 85 -94. doi:10.1109/IWAR.1999.803809.

[9] Maier-Hein L, Franz AM, Fangerau M, Schmidt M, Seitel A, Mersmann $\mathrm{S}$, et al. Towards mobile augmented reality for on-patient visualization of medical images. In: Bildverarbeitung für die Medizin. Informatik Aktuell; Springer. ISBN 978-3-540-19335-4; 2011, p. 389-93.

5 [10] Suenaga H, Hoang Tran H, Liao H, Masamune K, Dohi T, Hoshi K, et al. Real-time in situ three-dimensional integral videography and surgical navigation using augmented reality: a pilot study. International Journal of Oral Science 2013;(2):98-102.

[11] Blum T, Kleeberger V, Bichlmeier C, Navab N. mirracle: Augmented reality in-situ visualization of human anatomy using a magic mirror. In: VR, 2012 IEEE. 2012, p. 169-70. doi:10.1109/VR.2012.6180909.

[12] Meng M, Fallavollita P, Blum T, Eck U, Sandor C, Weidert S, et al. Kinect for interactive ar anatomy learning. In: Mixed and Augmented Reality (ISMAR), 2013 IEEE International Symposium on. 2013, p. 277-8. doi:10.1109/ISMAR.2013.6671803.

[13] Stefan P, Wucherer P, Oyamada Y, Ma M, Schoch A, Kanegae $M$, et al. An ar edutainment system supporting bone anatomy learning. In: Virtual Reality (VR), 2014 iEEE. 2014, p. 113-4. doi:10.1109/VR.2014.6802077.

[14] Mercier-Ganady J, Lotte F, Loup-escande E, Marchal M, Lecuyer A. The mind-mirror: See your brain in action in your head using eeg and augmented reality. In: Virtual Reality (VR), 2014 iEEE. 2014, p. 33-8. doi:10.1109/VR.2014.6802047.

[15] Macedo MC, Apolinario AL, Souza AC, Giraldi GA. A semi-automatic markerless augmented reality approach for on-patient volumetric medical data visualization. In: Virtual and Augmented Reality (SVR), 2014 XVI Symposium on. 2014, p. 63-70. doi:10.1109/SVR.2014.29.

[16] Macedo M, Almeida C, Souza A, Silva J, Apolinario A, Giraldi G. A Markerless Augmented Reality Environment for Medical Data Visualization. In: Workshop de Informatica Medica. WIM; Brazil; 2014,

[17] Macedo M, Apolinario A, Souza AC, Giraldi GA. High-Quality OnPatient Medical Data Visualization in a Markerless Augmented Reality Environment. Journal on 3D Interactive Systems 2014;5:41-52.

[18] Sandor C, Cunningham A, Dey A, Mattila VV. An augmented reality xray system based on visual saliency. In: ISMAR. IEEE Computer Society; 2010, p. 27-36. doi:10.1109/ISMAR.2010.5643547.

[19] Avery B, Sandor C, Thomas B. Improving spatial perception for augmented reality x-ray vision. In: Virtual Reality Conference, 2009. VR 2009. IEEE. 2009, p. 79-82. doi:10.1109/VR.2009.4811002.

[20] Mendez E, Feiner S, Schmalstieg D. Focus and context in mixed reality by modulating first order salient features. SG'10; Berlin, Heidelberg: Springer-Verlag. ISBN 3-642-13543-9, 978-3-642-13543-9; 2010, p. 232-43.

[21] Kalkofen D, Veas E, Zollmann S, Steinberger M, Schmalstieg D. Adaptive ghosted views for augmented reality. In: Mixed and Augmented Reality (ISMAR), 2013 IEEE International Symposium on. Adelaide, SA, Australia: IEEE Computer Society; 2013, p. 1-9. doi:10.1109/ISMAR.2013.6671758.

[22] Schall G, Mendez E, Kruijff E, Veas E, Junghanns S, Reitinger B, et al. Handheld augmented reality for underground infrastructure visualization. Personal Ubiquitous Comput 2009;13(4):281-91. doi:10.1007/s00779008-0204-5.

[23] Chen J, Granier X, Lin N, Peng Q. On-line visualization of underground structures using context features. In: Proceedings of the 17th ACM Symposium on Virtual Reality Software and Technology. VRST '10; New York, NY, USA: ACM. ISBN 978-1-4503-0441-2; 2010, p. 167-70. doi: $10.1145 / 1889863.1889898$.

[24] Padilha A, Rolim C, Teichrieb V. The ghosting technique applied to augmented reality visualization. In: Virtual and Augmented Reality (SVR), 2013 XV Symposium on. 2013, p. 159-66. doi:10.1109/SVR.2013.36.

[25] Mendez E, Schmalstieg D. Importance masks for revealing occluded objects in augmented reality. In: Proceedings of the 16th ACM Symposium on Virtual Reality Software and Technology. VRST '09; New York, NY, USA: ACM. ISBN 978-1-60558-869-8; 2009, p. 247-8.

5 [26] Lee CH, Varshney A, Jacobs DW. Mesh saliency. ACM Trans Graph 2005;24(3):659-66. doi:10.1145/1073204.1073244.

[27] Bruckner S, Grimm S, Kanitsar A, Gröller ME. Illustrative contextpreserving volume rendering. In: Proceedings of the Seventh Joint Eurographics / IEEE VGTC Conference on Visualization. EUROVIS'05; Airela-Ville, Switzerland, Switzerland: Eurographics Association. ISBN 3905673-19-3; 2005, p. 69-76. doi:10.2312/VisSym/EuroVis05/069-076.

[28] Bruckner S, Grimm S, Kanitsar A, Groller M. Illustrative context-preserving exploration of volume data. Visualization and 
Computer Graphics, IEEE Transactions on 2006;12(6):1559-69. 1135 doi:10.1109/TVCG.2006.96.

9] Sikachev P, Rautek P, Bruckner S, Gröller ME. Dynamic focus+context 1137 for volume rendering. In: Proceedings of Vision, Modeling and Visual- 1138 ization 2010. University of Siegen, Siegen, Germany; 2010, p. 331-8. $\quad 1139$ Kruger J, Schneider J, Westermann R. Clearview: An interac- 1140 tive context preserving hotspot visualization technique. Visualiza- 1141 tion and Computer Graphics, IEEE Transactions on 2006;12(5):941-8. 1142 doi:10.1109/TVCG.2006.124.

1] Kirmizibayrak C, Wakid M, Yim Y, Hristov D, Hahn JK. Interactive fo- 1144 cus + context medical data exploration and editing. Computer Animation 1145 and Virtual Worlds 2014;25(2):129-41. doi:10.1002/cav.1538.

2] Röttger D, Merhof D, Müller S. The BundleExplorer: A Fo- 1147 cus and Context Rendering Framework for Complex Fiber Distribu- 1148 tions. In: Proceedings of the Eurographics Conference on Visual 1149 Computing for Biology and Medicine. EG VCBM'12; 2012, p. 1-8. 1150 doi:10.2312/VCBM/VCBM12/001-008.

3] Gasteiger R, Neugebauer M, Beuing O, Preim B. The flowlens: A focus- 1152 and-context visualization approach for exploration of blood flow in cere- 1153 bral aneurysms. Visualization and Computer Graphics, IEEE Transac- 1154 tions on 2011;17(12):2183-92. doi:10.1109/TVCG.2011.243.

4] Abellan P, Puig A, Ayala D. Focus + context rendering of structured biomedical data. In: VCBM'08. 2008, p. 109-16.

Schulte zu Berge C, Baust M, Kapoor A, Navab N Predicate- 1158 based focus-and-context visualization for 3d ultrasound. Visualiza- 1159 tion and Computer Graphics, IEEE Transactions on 2014;PP(99):1-. 1160 doi:10.1109/TVCG.2014.2346317.

36] Kersten-Oertel M, Jannin P, Collins D. Dvv: A taxonomy for 1162 mixed reality visualization in image guided surgery. Visualization 1163 and Computer Graphics, IEEE Transactions on 2012;18(2):332-52. 1164 doi:10.1109/TVCG.2011.50.

37] Lerotic M, Chung AJ, Mylonas G, Yang GZ. Pq-space based 1166 non-photorealistic rendering for augmented reality. In: Proceedings 1167 of the 10th International Conference on Medical Image Computing 1168 and Computer-assisted Intervention. MICCAI'07; Berlin, Heidelberg: 1169 Springer-Verlag. ISBN 3-540-75758-9, 978-3-540-75758-0; 2007, p. 1170 102-9.

\author{
1171
}

] Pratt P, Mayer E, Vale J, Cohen D, Edwards E, Darzi A, et al. An 1172 effective visualisation and registration system for image-guided robotic 1173 $\begin{array}{ll} & \\ \text { doi:10.1007/s11701-011-0334-z. } & \\ 1174\end{array}$ Bichlmeier C, Heining S, Feuerstein M, Navab N. The virtual 1176 mirror: A new interaction paradigm for augmented reality environ- 1177 ments. Medical Imaging, IEEE Transactions on 2009;28(9):1498-510. 1178 doi:10.1109/TMI.2009.2018622.

40] Kersten-Oertel M, Chen SS, Collins D. An evaluation of depth enhancing 1180 perceptual cues for vascular volume visualization in neurosurgery. Visu- 1181 alization and Computer Graphics, IEEE Transactions on 2014;20(3):391- 1182 403. doi:10.1109/TVCG.2013.240.

41] Steenblik RA. The chromostereoscopic process: A novel single 1184 image stereoscopic process. vol. 0761. 1987, p. 27-34. URL: 1185 http://dx.doi.org/10.1117/12.940117. doi:10.1117/12.940117. 1186

42] Viola P, Jones MJ. Robust real-time face detection. Int J Comput Vision 1187 2004;57(2):137-54. doi:10.1023/B:VISI.0000013087.49260.fb.

43] Tomasi C, Manduchi R. Bilateral filtering for gray and color images. In: 1189 Computer Vision, 1998. Sixth International Conference on. 1998, p. 8391190 -46. doi:10.1109/ICCV.1998.710815.

44] Burt PJ. Fast filter transform for image processing. Computer Graphics and Image Processing 1981;16(1):20 — 51. 1193 doi:http://dx.doi.org/10.1016/0146-664X(81)90092-7.

51. 1193 Adelson EH, Anderson CH, Bergen JR, Burt PJ, Ogden JM. Pyramid 1195
methods in image processing. RCA Engineer 1984;29(6):33-41. Izadi S, Kim D, Hilliges O, Molyneaux D, Newcombe R, Kohli P, et al. ${ }_{1197}^{1196}$ Kinectfusion: real-time $3 \mathrm{~d}$ reconstruction and interaction using a moving 1198 depth camera. In: Proceedings of the 24th Annual ACM Symposium on 1199 User Interface Software and Technology. UIST '11; USA: ACM. ISBN 1200 978-1-4503-0716-1; 2011, p. 559-68. doi:10.1145/2047196.2047270. 1201

47] Curless B, Levoy M. A volumetric method for building complex mod- 1202 els from range images. In: Proceedings of the 23rd Annual Confer- 1203 ence on Computer Graphics and Interactive Techniques. SIGGRAPH '96; 1204 New York, NY, USA: ACM. ISBN 0-89791-746-4; 1996, p. 303-12. 1205 [72] doi:10.1145/237170.237269.

[48] Meister S, Izadi S, Kohli P, Hämmerle M, Rother C, Kondermann D. When can we use kinectfusion for ground truth acquisition? In: IROS. IEEE Computer Society; 2012,.

49] Rusinkiewicz S, Levoy M. Efficient variants of the icp algorithm. In: Third International Conference on 3D Digital Imaging and Modeling (3DIM). 2001, p. 145-52.

[50] Newcombe RA, Izadi S, Hilliges O, Molyneaux D, Kim D, Davison AJ, et al. Kinectfusion: Real-time dense surface mapping and tracking. In: Mixed and Augmented Reality (ISMAR), 2011 10th IEEE International Symposium on. 2011, p. 127-36. doi:10.1109/ISMAR.2011.6092378.

51] Fanelli G, Weise T, Gall J, Gool LV. Real time head pose estimation from consumer depth cameras. In: Proceedings of the 33rd International Conference on Pattern Recognition. DAGM'11; Berlin, Heidelberg: SpringerVerlag. ISBN 978-3-642-23122-3; 2011, p. 101-10.

[52] Macedo M, Apolinario A, Souza A. A robust real-time face tracking using head pose estimation for a markerless ar system. In: Virtual and Augmented Reality (SVR), 2013 XV Symposium on. 2013, p. 224-7. doi:10.1109/SVR.2013.12.

53] Hadwiger M, Kniss JM, Rezk-salama C, Weiskopf D, Engel K. Real-time Volume Graphics. USA: A. K. Peters, Ltd.; 2006. ISBN 1568812663

54] Hadwiger M, Ljung P, Salama CR, Ropinski T. Advanced illumination techniques for gpu-based volume raycasting. SIGGRAPH '09; New York, NY, USA: ACM; 2009, p. 2:1-2:166

[55] Sigg C, Hadwiger M. Fast third-order texture filtering. In: Pharr M, editor. GPU Gems 2. Addison-Wesley; 2005, p. 313-29.

[56] Ruijters D, ter Haar Romeny BM, Suetens P. Efficient gpu-based texture interpolation using uniform b-splines. J Graphics Tools 2008;13(4):61-9.

57] Li W, Mueller K, Kaufman A. Empty space skipping and occlusion clipping for texture-based volume rendering. In: VIS 2003. IEEE Computer Society; 2003, p. 317-24.

[58] Engel K, Kraus M, Ertl T. High-quality pre-integrated volume rendering using hardware-accelerated pixel shading. HWWS '01; New York, NY, USA: ACM. ISBN 1-58113-407-X; 2001, p. 9-16.

[59] Blinn JF. Models of light reflection for computer synthesized pictures. SIGGRAPH '77; New York, NY, USA: ACM; 1977, p. 192-8.

60] Ruijters D, Thévenaz P. Gpu prefilter for accurate cubic b-spline interpolation. Comput J 2012;55(1):15-20. doi:10.1093/comjnl/bxq086.

[61] Wu ST, Yauri Vidalon J, Souza Loos W, Coan A. Query tools for interactive exploration of $3 \mathrm{~d}$ neuroimages: Cropping, probe and lens. In: Graphics, Patterns and Images (SIBGRAPI), 2013 26th SIBGRAPI - Conference on. 2013, p. 250-7. doi:10.1109/SIBGRAPI.2013.42.

[62] Otsu N. A Threshold Selection Method from Gray-level Histograms. SMC 1979;9(1):62-6. doi:10.1109/tsmc.1979.4310076.

[63] Suzuki S. Topological structural analysis of digitized binary images by border following. CVGIP 1985;30(1):32 - 46 . doi:http://dx.doi.org/10.1016/0734-189X(85)90016-7.

[64] Rusu R, Cousins S. 3d is here: Point cloud library (pcl). In: ICRA. IEEE Computer Society; 2011, p. 1 -4. doi:10.1109/ICRA.2011.5980567.

[65] Cruz L, Lucio D, Velho L. Kinect and rgbd images: Challenges and applications. In: Graphics, Patterns and Images Tutorials (SIBGRAPI-T), 2012 25th SIBGRAPI Conference on. 2012, p. 36-49. doi:10.1109/SIBGRAPI-T.2012.13.

[66] Visible human data. http://www.nlm.nih.gov/research /visible/; 2014. Accessed 22 September 2014.

67] Volume library. http://www9.informatik.unierlangen.de/External/vollib/; 2014. Acessed 22 September 2014.

[68] Osirix. http://www.osirix-viewer.com/datasets/; 2015. Acessed 26 May 2015.

[69] Akenine-Moller T, Moller T, Haines E. Real-Time Rendering. 2nd ed.; Natick, MA, USA: A. K. Peters, Ltd.; 2002. ISBN 1568811829.

70] Peasley B, Birchfield S. Replacing projective data association with lucas-kanade for kinectfusion. In: Robotics and Automation (ICRA), 2013 IEEE International Conference on. 2013, p. 638-45. doi:10.1109/ICRA.2013.6630640.

71] Lucas BD, Kanade T. An iterative image registration technique with an application to stereo vision. In: Proceedings of the 7th International Joint Conference on Artificial Intelligence - Volume 2. IJCAI'81; San Francisco, CA, USA: Morgan Kaufmann Publishers Inc.; 1981, p. 674-9.

72] Horn BK, Schunck BG. Determining optical flow. Artificial Intel- 
1206 ligence 1981;17(1-3):185 - 203. doi:http://dx.doi.org/10.1016/00041207 3702(81)90024-2.

1208 [73] Zollhöfer M, Nießner M, Izadi S, Rhemann C, Zach C, Fisher M, et al 1209 Real-time non-rigid reconstruction using an rgb-d camera. ACM Trans$1210 \quad$ actions on Graphics (TOG) 2014;33(4).

1211 [74] Souza ACS, Macedo MCF, Apolinário Jr. AL. Multi-frame adaptive 1212 non-rigid registration for markerless augmented reality. In: Proceed1213 ings of the 13th ACM SIGGRAPH International Conference on Virtual1214 Reality Continuum and Its Applications in Industry. VRCAI '14; New 1215 York, NY, USA: ACM. ISBN 978-1-4503-3254-5; 2014, p. 7-16. 1216 doi:10.1145/2670473.2670486.

1217 [75] Nießner M, Zollhöfer M, Izadi S, Stamminger M. Real-time 3d recon1218 struction at scale using voxel hashing. ACM Transactions on Graphics 1219 (TOG) 2013; 